Supporting Information for

\title{
Linear Biazulene Isomers: Effects of Molecular and Packing Structure on Optoelectronic and Charge-transport Properties
}

Yusuke Shibuya, ${ }^{+}$Kazuhiro Aonuma, ${ }^{*}$ Tomohiro Kimura, ${ }^{+}$Takuro Kaneko, ${ }^{*}$ Wataru Fujiwara, ${ }^{+}$Yuji Yamaguchi, ${ }^{*}$ Daisuke Kumaki, ${ }^{\S}$ Shizuo Tokito, ${ }^{\S}$ and Hiroshi Katagiri *

Hraduate School of Science and Engineering, Yamagata University, 4-3-16 Jonan, Yonezawa, Yamagata 992-8510, Japan

${ }^{\S}$ Graduate School of Organic Materials Science, Yamagata University, 4-3-16 Jonan, Yonezawa, Yamagata, 992-8510, Japan

\section{Contents}

1. Syntheses of 6-bromoazulene, 2-chloroazulene, and azulene-2-boronic acid pinacol ester

2. ${ }^{1} \mathrm{H}$ and ${ }^{13} \mathrm{C}$ NMR and HRMS spectra for all the target compounds

3. Cartesian coordinates and energies of all optimized structures

4. X-ray crystallographic data and structure refinement for all the target compounds

5. FET characteristics of biazulene isomers 


\section{Syntheses of 6-bromoazulene, 2-chloroazulene, and azulene-2-boronic acid pinacol ester}
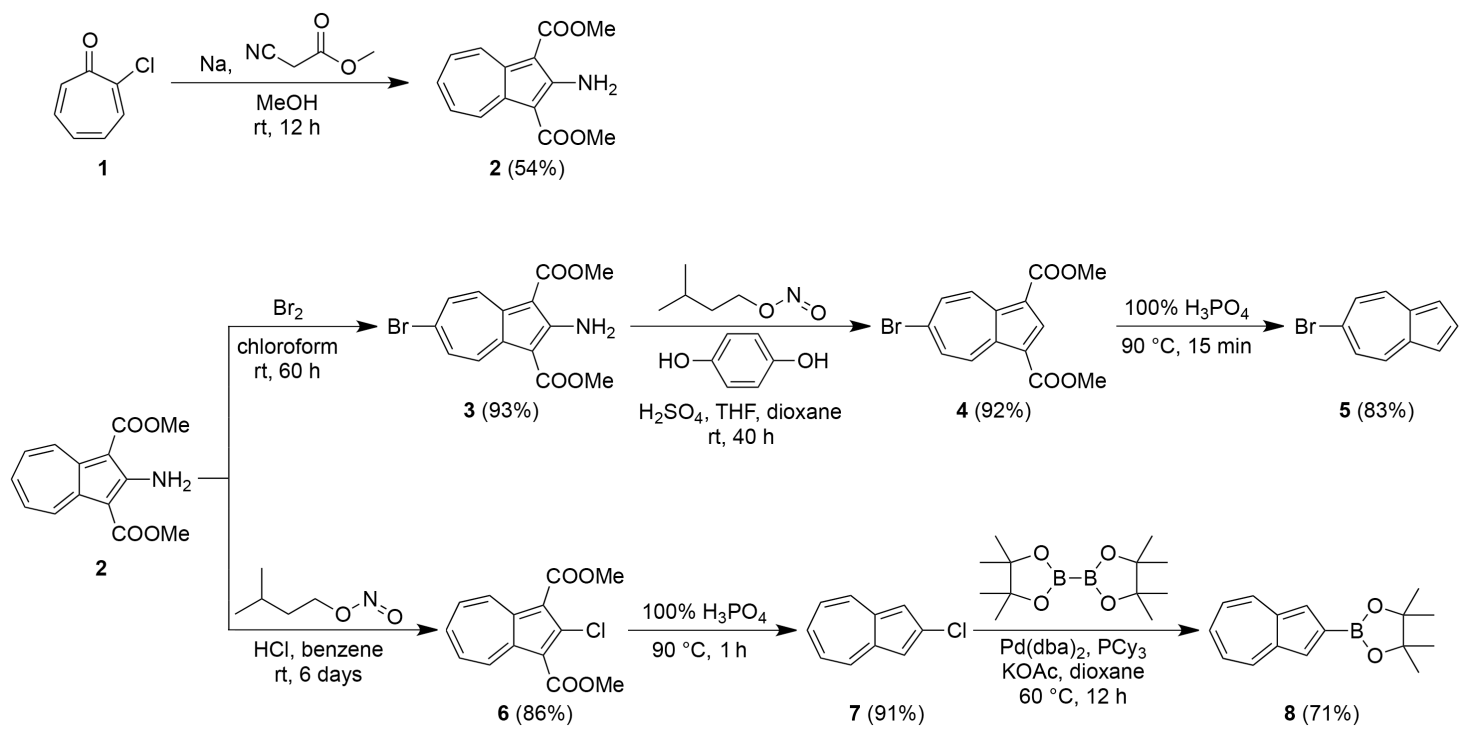

Scheme S1. Synthetic route to 6-bromoazulene 5, 2-chloroazulene 7, and azulene-2-boronic acid pinacol ester $\mathbf{8}$.

\section{Dimethyl 2-aminoazulene-1,3-dicarboxylate 2:}<smiles></smiles>

1

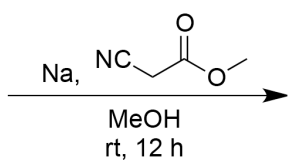

$\mathrm{rt}, 12 \mathrm{~h}$

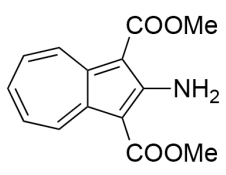

2 (54\%)

Sodium methoxide was prepared by adding sodium (7.72 g, $0.336 \mathrm{~mol})$ to dry $\mathrm{MeOH}(240 \mathrm{~mL})$ gradually while cooling at $0{ }^{\circ} \mathrm{C}$ in a nitrogen atmosphere. Methyl cyanoacetate $(59 \mathrm{~mL}, 0.672 \mathrm{~mol})$ was added to the solution of sodium methoxide at $0{ }^{\circ} \mathrm{C}$, and then 2-chlorotropone ( $23.6 \mathrm{~g}, 0.168 \mathrm{~mol}$ ) that was dissolved in dry $\mathrm{MeOH}(240 \mathrm{~mL})$ was added dropwise at $0{ }^{\circ} \mathrm{C}$, followed by stirring at $25^{\circ} \mathrm{C}$ for $12 \mathrm{~h}$. The reaction mixture was concentrated in vacuo, dissolved in chloroform (350 mL), and filtered to remove inorganic salts. After the filtrate was concentrated under reduced pressure, the obtained crude product was suspended in cold EtOH $(100 \mathrm{~mL})$. The suspension was filtered, washed with cold EtOH to remove excess amount of methyl cyanoacetate, and dried in vacuo to give 2aminoazulene-1,3-dicarboxylate $2(23.3 \mathrm{~g}, 54 \%)$ as orange powder. ${ }^{1} \mathrm{H} \mathrm{NMR}\left(\mathrm{CDCl}_{3}\right): \delta(\mathrm{ppm}): 9.12$ (d, $J=10.5 \mathrm{~Hz}, 2 \mathrm{H}), 7.77$ (s, 2H), 7.55 (dd, $J=9.8,10.5 \mathrm{~Hz}, 2 \mathrm{H}), 7.45$ (t, $J=9.8 \mathrm{~Hz}, 1 \mathrm{H}), 3.99$ (s, $6 \mathrm{H})$. 


\section{2-Amino-6-bromoazulene-1,3-dicarboxylic acid dimethyl ester 3:}

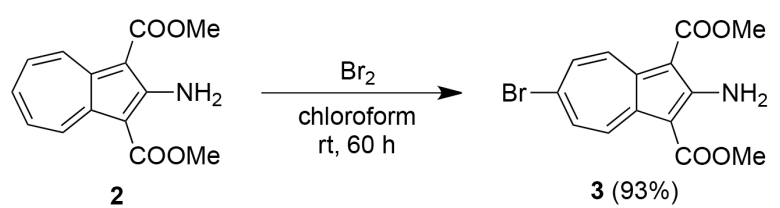

Bromine $(0.30 \mathrm{~mL}, 5.69 \mathrm{mmol})$ was added dropwise to a stirred solution of dimethyl 2-aminoazulene1,3-dicarboxylate $2(1.23 \mathrm{~g}, 4.75 \mathrm{mmol})$ in chloroform $(50 \mathrm{~mL})$ at $0{ }^{\circ} \mathrm{C}$. Then the mixture was stirred at $25^{\circ} \mathrm{C}$ for $60 \mathrm{~h}$. The reaction mixture was quenched by adding saturated sodium hydrogen sulfite solution. After the organic phase was separated, the aqueous phase was extracted twice with chloroform $(30 \mathrm{~mL})$. The organic phases were combined and washed sequentially with water and saturated sodium hydrogen carbonate solution. After drying over sodium sulfate, the solution was concentrated in vacuo to produce 2-amino-6-bromoazulene-1,3-dicarboxylic acid dimethyl ester 3 $(1.48 \mathrm{~g}, 93 \%)$ as orange powder. ${ }^{1} \mathrm{H}$ NMR $\left(\mathrm{CDCl}_{3}\right): \delta(\mathrm{ppm}): 8.81$ (d, $\left.J=11.8 \mathrm{~Hz}, 2 \mathrm{H}\right), 7.82-7.79(\mathrm{~m}$, $4 \mathrm{H}), 3.99(\mathrm{~s}, 6 \mathrm{H})$.

\section{Dimethyl 6-bromoazulene-1,3-dicarboxylate 4:}

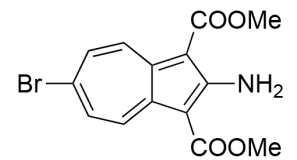

3

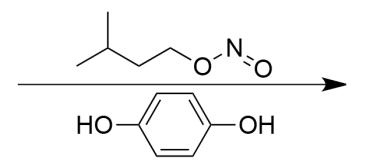

$\mathrm{H}_{2} \mathrm{SO}_{4}$, THF, dioxane

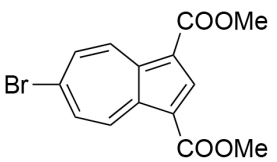

$4(92 \%)$

2-Amino-6-bromoazulene-1,3-dicarboxylic acid dimethyl ester $3(5.82 \mathrm{~g}, 17.2 \mathrm{mmol})$ and hydroquinone $(1.63 \mathrm{~g}, 14.8 \mathrm{mmol})$ were added to dioxane $(440 \mathrm{~mL})$ and stirred in a nitrogen atmosphere. Then sulfuric acid $(1.52 \mathrm{~g}, 15.6 \mathrm{mmol})$ in tetrahydrofuran $(14.0 \mathrm{~mL})$ was added to the solution. Furthermore, a solution of hydroquinone $(16.3 \mathrm{~g}, 0.148 \mathrm{~mol})$ in dioxane $(290 \mathrm{~mL})$ and a solution of isoamyl nitrite $(18.2 \mathrm{~g}, 0.155 \mathrm{~mol})$ in dioxane $(290 \mathrm{~mL})$ were added dropwise at the same time over a period of $90 \mathrm{~min}$, and the mixture was then stirred at $25{ }^{\circ} \mathrm{C}$ for $40 \mathrm{~h}$. Subsequently, the concentrated crude product was dissolved in tetrahydrofuran, and then adsorbed on silica gel and purified by column chromatography (chloroform) to afford dimethyl 6-bromoazulene-1,3dicarboxylate 4 (5.10 g, 92\%) as red crystals. ${ }^{1} \mathrm{H}$ NMR $\left(\mathrm{CDCl}_{3}\right): \delta(\mathrm{ppm}): 9.50(\mathrm{~d}, J=11.3 \mathrm{~Hz}, 2 \mathrm{H})$, $8.82(\mathrm{~s}, 1 \mathrm{H}), 8.06(\mathrm{~d}, J=11.3 \mathrm{~Hz}, 2 \mathrm{H}), 3.96(\mathrm{~s}, 6 \mathrm{H})$. 


\section{6-Bromoazulene 5:}

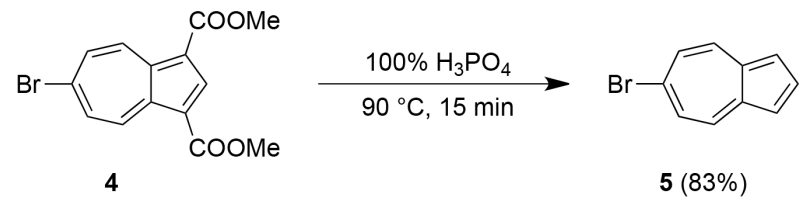

Phosphorus (V) oxide (100 g, $0.352 \mathrm{~mol}$ ) was added slowly to $85 \%$ phosphoric acid ( $250 \mathrm{~g}, 2.17 \mathrm{~mol})$ in an ice bath with stirring to prepare $100 \%$ phosphoric acid. The supernatant, from which precipitates were removed from $100 \%$ phosphoric acid, was heated to $90{ }^{\circ} \mathrm{C}$ in a nitrogen atmosphere. Then, dimethyl 6-bromoazulene-1,3-dicarboxylate 4 (2.00 g, $6.19 \mathrm{mmol})$ was added and stirred vigorously for $15 \mathrm{~min}$. After quenching the reaction solution by pouring it into ice water $(600 \mathrm{~mL}), n$-hexane $(600$ $\mathrm{mL}$ ) was added and stirred to dissolve the reactants. After the organic phases was separated, the aqueous phase was extracted with $n$-hexane $(600 \mathrm{~mL})$. The resulting organic phases were combined, washed with a saturated sodium hydrogen carbonate solution $(100 \mathrm{~mL})$, and dried over sodium sulfate. The solution was then concentrated to $100 \mathrm{~mL}$ with a rotary evaporator and purified by column chromatography ( $n$-hexane) to provide 6-bromoazulene $5(1.06 \mathrm{~g}, 83 \%)$ as purple crystals. ${ }^{1} \mathrm{H}$ NMR $\left(\mathrm{CDCl}_{3}\right): \delta(\mathrm{ppm}): 8.05(\mathrm{~d}, J=11.0 \mathrm{~Hz}, 2 \mathrm{H}), 7.92(\mathrm{t}, J=4.0 \mathrm{~Hz}, 1 \mathrm{H}), 7.51(\mathrm{~d}, J=11.0 \mathrm{~Hz}, 2 \mathrm{H}), 7.42$ $(\mathrm{d}, J=4.0 \mathrm{~Hz}, 2 \mathrm{H})$.

\section{Dimethyl 2-chloroazulene-1,3-dicarboxylate 6:}

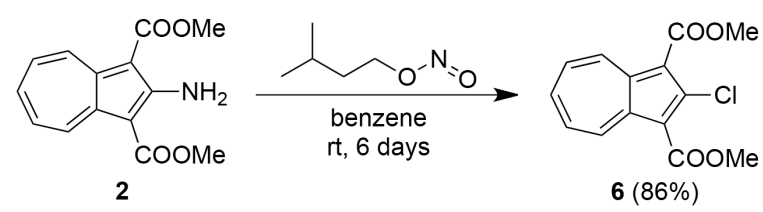

Dimethyl 2-aminoazulene-1,3-dicarboxylate $2(2.28 \mathrm{~g}, 8.79 \mathrm{mmol})$ was dissolved in dry benzene (70 $\mathrm{mL}$ ) and cooled to $5{ }^{\circ} \mathrm{C}$, and then $\mathrm{HCl}$ gas that was generated by adding concentrated sulfuric acid dropwise to sodium chloride was bubbled over for $5 \mathrm{~h}$ while stirring at $5{ }^{\circ} \mathrm{C}$. After a solution of isoamyl nitrite $(1.13 \mathrm{~g}, 9.65 \mathrm{mmol})$ in dry benzene $(5 \mathrm{~mL})$ was added dropwise during a period of $15 \mathrm{~min}$, the reaction temperature was increased to $25^{\circ} \mathrm{C}$, and the mixture was stirred for 6 days. The reaction mixture was poured into water $(80 \mathrm{~mL})$ to quench the reaction, and the organic phase was separated. The organic phase was washed 2 times with water $(80 \mathrm{~mL})$, dried over sodium sulfate, and then, concentrated in vacuo. The crude product was recrystallized from EtOH to provide dimethyl 2chloroazulene-1,3-dicarboxylate $6(2.10 \mathrm{~g}, 86 \%)$ as red crystals. ${ }^{1} \mathrm{H}$ NMR $\left(\mathrm{CDCl}_{3}\right): \delta(\mathrm{ppm}): 9.54$ (d, $J=10.3 \mathrm{~Hz}, 2 \mathrm{H}), 7.95(\mathrm{t}, J=9.5 \mathrm{~Hz}, 1 \mathrm{H}), 7.76(\mathrm{dd}, J=9.5,10.3 \mathrm{~Hz}, 2 \mathrm{H}), 4.03(\mathrm{~s}, 6 \mathrm{H}) ;{ }^{13} \mathrm{C} \mathrm{NMR}$ $\left(\mathrm{CDCl}_{3}\right): \delta(\mathrm{ppm}): 164.80,143.52,141.87,140.40,138.20,131.26,115.17,51.68 ; \mathrm{IR}(\mathrm{KBr}): v=1716$ $(\mathrm{C}=\mathrm{O}) \mathrm{cm}^{-1}$; HRMS $\left(\mathrm{FD}^{+}\right): \mathrm{m} / z$ calcd. for $\mathrm{C}_{14} \mathrm{H}_{11} \mathrm{ClO}_{4}\left[M^{+}\right]: 278.03480$; found: 278.03459; elemental analysis calcd. (\%) for $\mathrm{C}_{14} \mathrm{H}_{11} \mathrm{ClO}_{4}$ : $\mathrm{C}$ 60.34, $\mathrm{H} 3.98$; found: $\mathrm{C} 60.07, \mathrm{H} 3.84$. 


\section{2-Chloroazulene 7:}

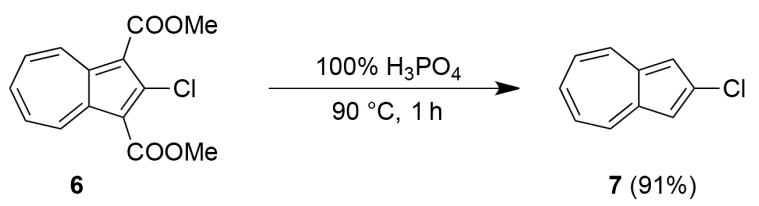

Phosphorus (V) oxide (150 g, $0.528 \mathrm{~mol})$ was added slowly to 85\% phosphoric acid (375 g, $3.25 \mathrm{~mol}$ ) in an ice bath with stirring to prepare $100 \%$ phosphoric acid. The supernatant, from which precipitates were removed from $100 \%$ phosphoric acid, was heated to $90{ }^{\circ} \mathrm{C}$ in a nitrogen atmosphere. Then, dimethyl 2-chloroazulene-1,3-dicarboxylate 6 (10.0 g, $35.9 \mathrm{mmol})$ was added and stirred vigorously for 1 hour. After quenching the reaction solution by pouring it into ice water $(1000 \mathrm{~mL}), n$-hexane $(500 \mathrm{~mL})$ was added and stirred to dissolve the reactants. After the organic phases was separated, the aqueous phase was extracted with $n$-hexane $(500 \mathrm{~mL})$. The resulting organic phases were combined, washed with a saturated sodium hydrogen carbonate solution $(500 \mathrm{~mL})$, and dried over sodium sulfate. The solution was then concentrated to $100 \mathrm{~mL}$ with a rotary evaporator and purified by column chromatography ( $n$-hexane) to provide 2-chloroazulene 7 (5.31 g, 91\%) as purple crystals. ${ }^{1} \mathrm{H}$ NMR (DMSO- $\left.d_{6}\right): \delta(\mathrm{ppm}): 8.40$ (d, $\left.J=9.5 \mathrm{~Hz}, 2 \mathrm{H}\right), 7.76(\mathrm{t}, J=10.0 \mathrm{~Hz}, 1 \mathrm{H}), 7.40-7.36(\mathrm{~m}, 4 \mathrm{H})$.

\section{Azulene-2-boronic acid pinacol ester 8:}

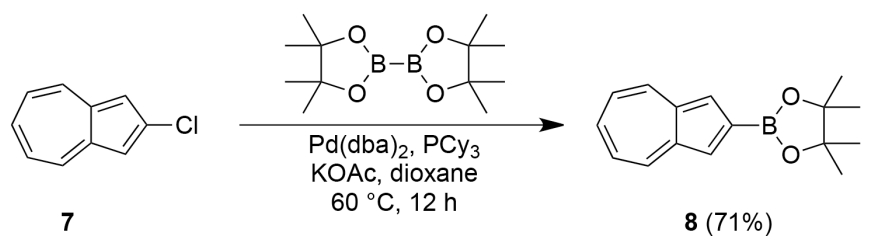

Bis (dibenzylideneacetone) palladium $(0)(0.20 \mathrm{~g}, 0.348 \mathrm{mmol})$ and tricyclohexylphosphine $(0.23 \mathrm{~g}$, $0.820 \mathrm{mmol})$ were stirred in dioxane $(70 \mathrm{~mL})$ at $25{ }^{\circ} \mathrm{C}$ for $30 \mathrm{~min}$ in a nitrogen atmosphere, then bis (pinacolato) diboron (4.32 g, $17.0 \mathrm{mmol})$, potassium acetate $(1.67 \mathrm{~g}, 17.0 \mathrm{mmol})$ and 2-chloroazulene $7(1.83 \mathrm{~g}, 11.3 \mathrm{mmol})$ were added and stirred at $60^{\circ} \mathrm{C}$ for 12 hours. The reaction mixture was poured into water $(500 \mathrm{~mL})$ for quenching, then extracted with dichloromethane $(500 \mathrm{~mL})$, and then the organic layer was dried over sodium sulfate. After the solution was concentrated, the crude product purified by column chromatography (dichloromethane $/ n$-hexane $=5 / 1$ ) to give azulene- 2 boronic acid pinacol ester 8 as blue powder (2.04 g, 71\%). ${ }^{1} \mathrm{H}$ NMR $\left(\mathrm{CDCl}_{3}\right): \delta(\mathrm{ppm}): 8.35(\mathrm{~d}, J=9.5 \mathrm{~Hz}, 2 \mathrm{H})$, $7.76(\mathrm{~s}, 2 \mathrm{H}), 7.58(\mathrm{t}, \mathrm{J}=10.0 \mathrm{~Hz}, 1 \mathrm{H}), 7.12(\mathrm{dd}, J=9.5,10.0 \mathrm{~Hz}, 2 \mathrm{H}), 1.40$ (s, 12H). 
2. ${ }^{1} \mathrm{H}$ and ${ }^{13} \mathrm{C}$ NMR and HRMS spectra for all the target compounds

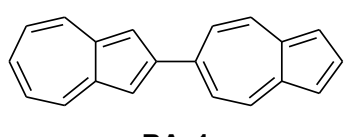

BAz1

(a)

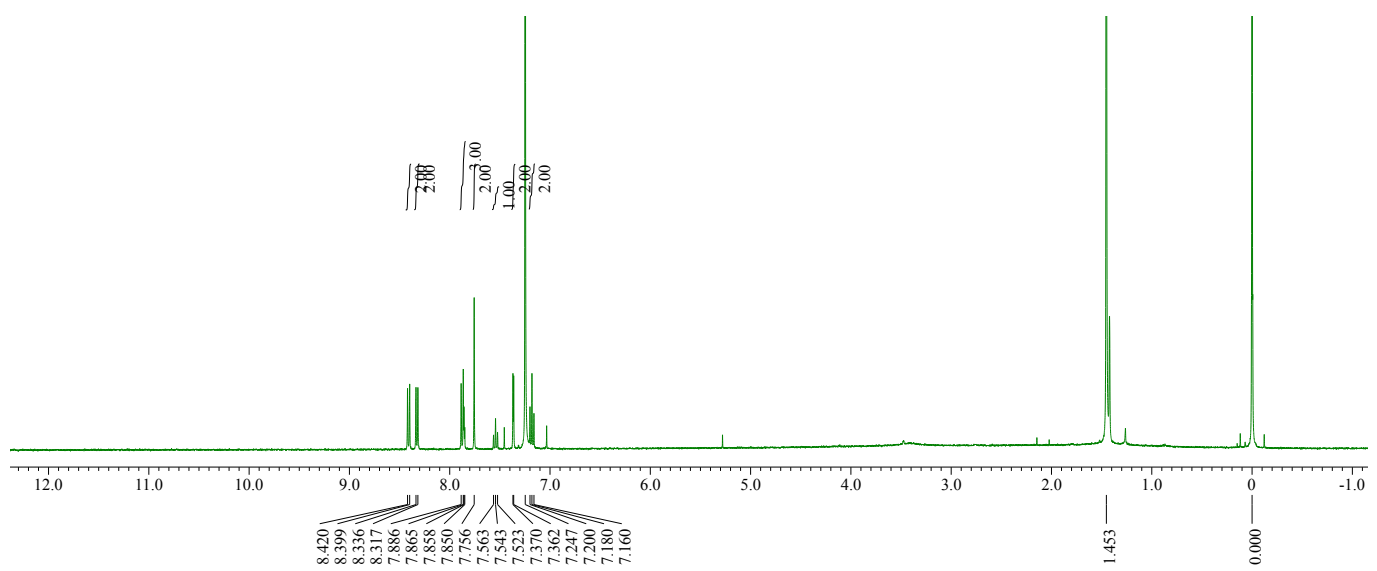

(b)

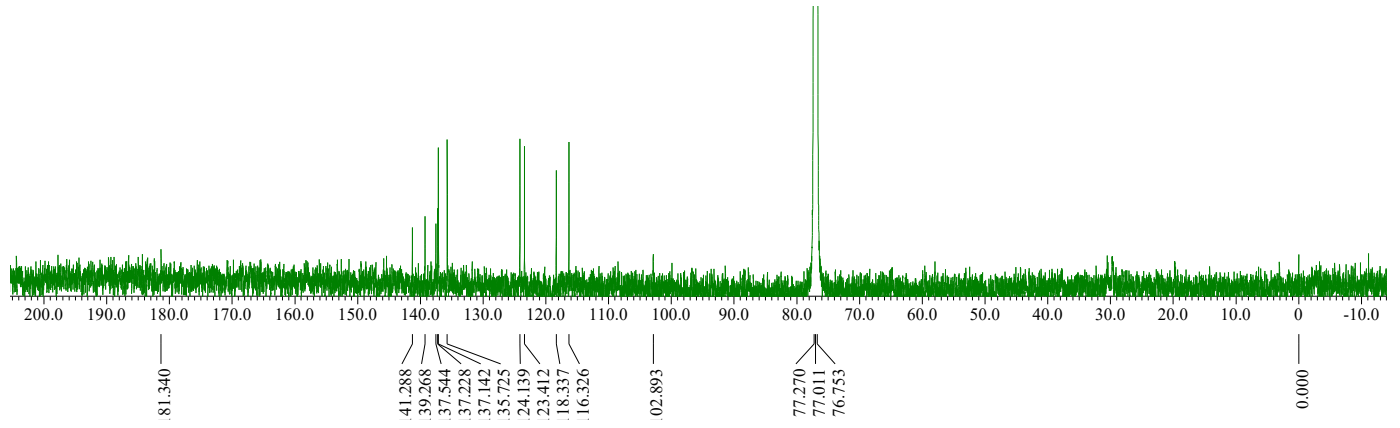

(c)

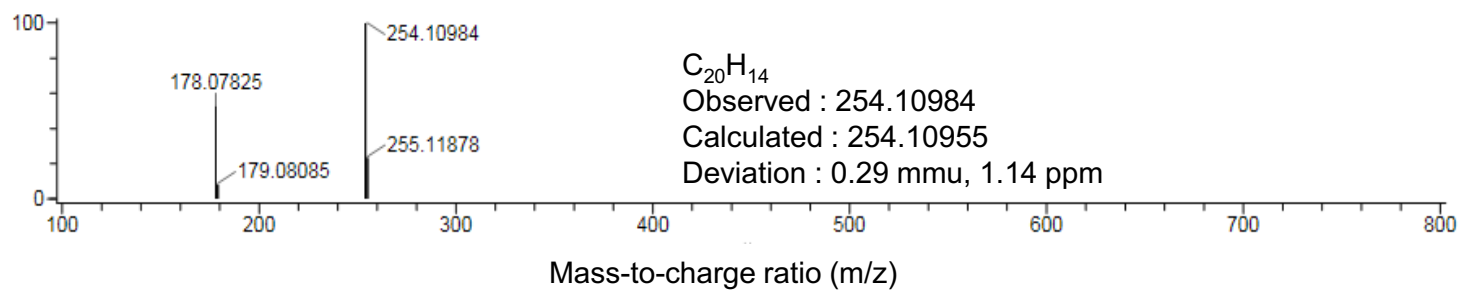

Figure S1. (a) ${ }^{1} \mathrm{H}$ NMR spectrum $\left(500 \mathrm{MHz}, \mathrm{CDCl}_{3}, 50^{\circ} \mathrm{C}\right)$, (b) ${ }^{13} \mathrm{C} \mathrm{NMR}$ spectrum $\left(125 \mathrm{MHz}, \mathrm{CDCl}_{3}\right.$, $50{ }^{\circ} \mathrm{C}$ ), and (c) FD-HRMS spectrum of BAz1. Anthracene [calcd for $\mathrm{C}_{14} \mathrm{H}_{10}\left(M^{+}\right): m / z=178.07825$ ] was used as an internal standard in HRMS analysis. 


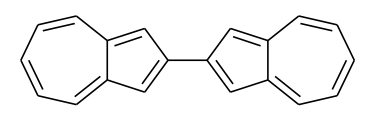

BAz2

(a)

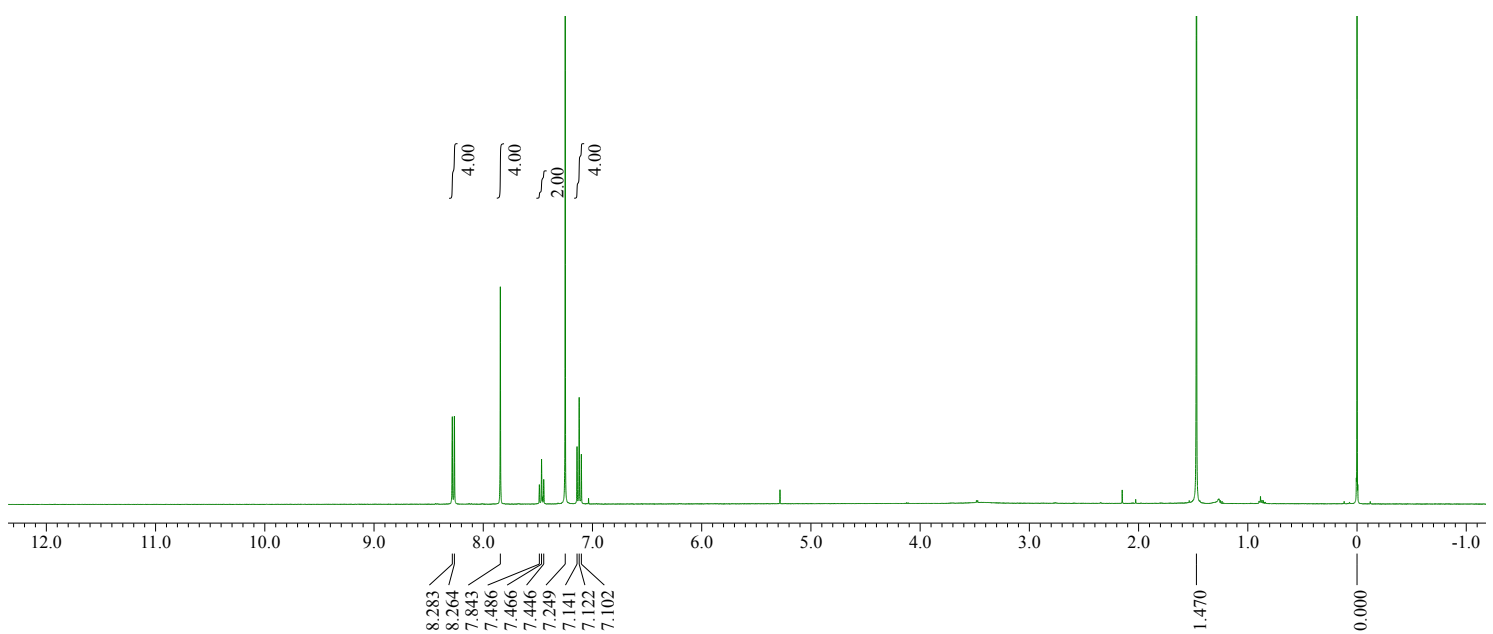

(b)

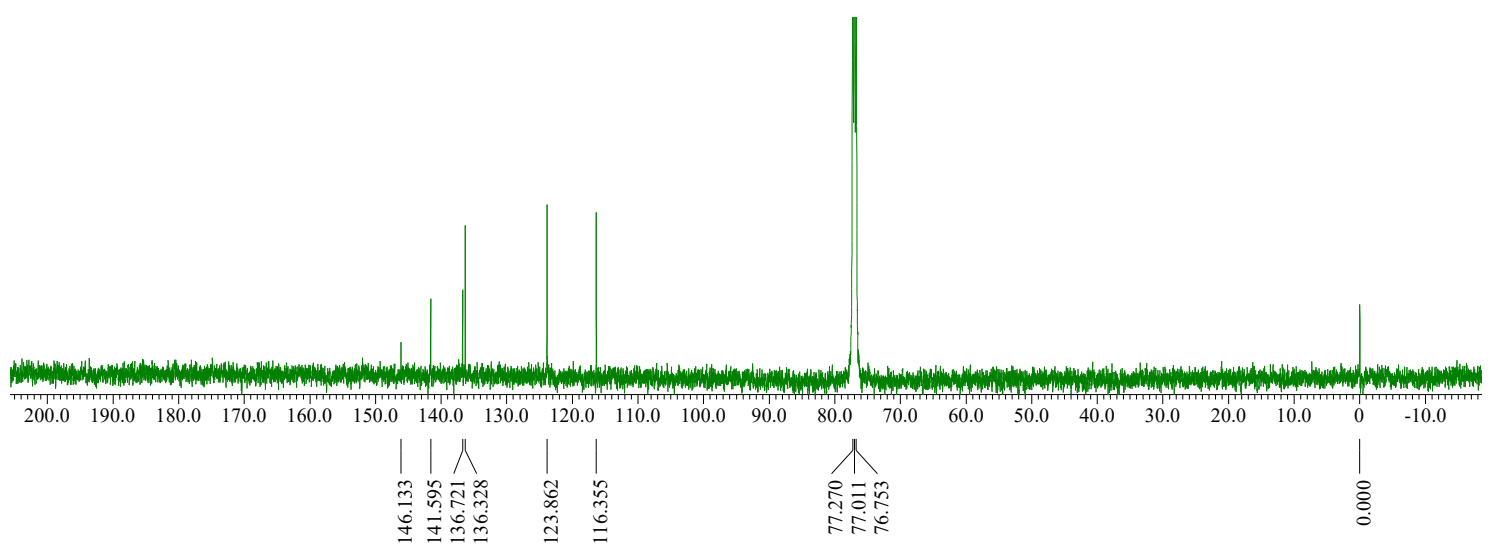

(c)

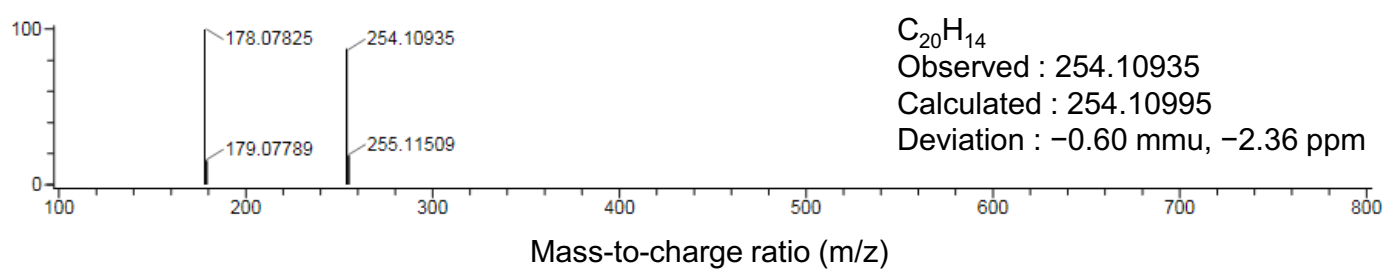

Figure S2. (a) ${ }^{1} \mathrm{H}$ NMR spectrum ( $500 \mathrm{MHz}, \mathrm{CDCl}_{3}, 50{ }^{\circ} \mathrm{C}$ ), (b) ${ }^{13} \mathrm{C} \mathrm{NMR}$ spectrum $\left(125 \mathrm{MHz}, \mathrm{CDCl}_{3}\right.$, $50{ }^{\circ} \mathrm{C}$ ), and (c) FD-HRMS spectrum of BAz2. Anthracene [calcd for $\mathrm{C}_{14} \mathrm{H}_{10}\left(M^{+}\right): m / z=178.07825$ ] was used as an internal standard in HRMS analysis. 


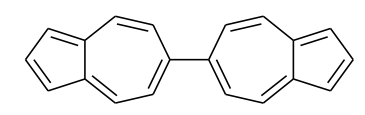

BAz3

(a)

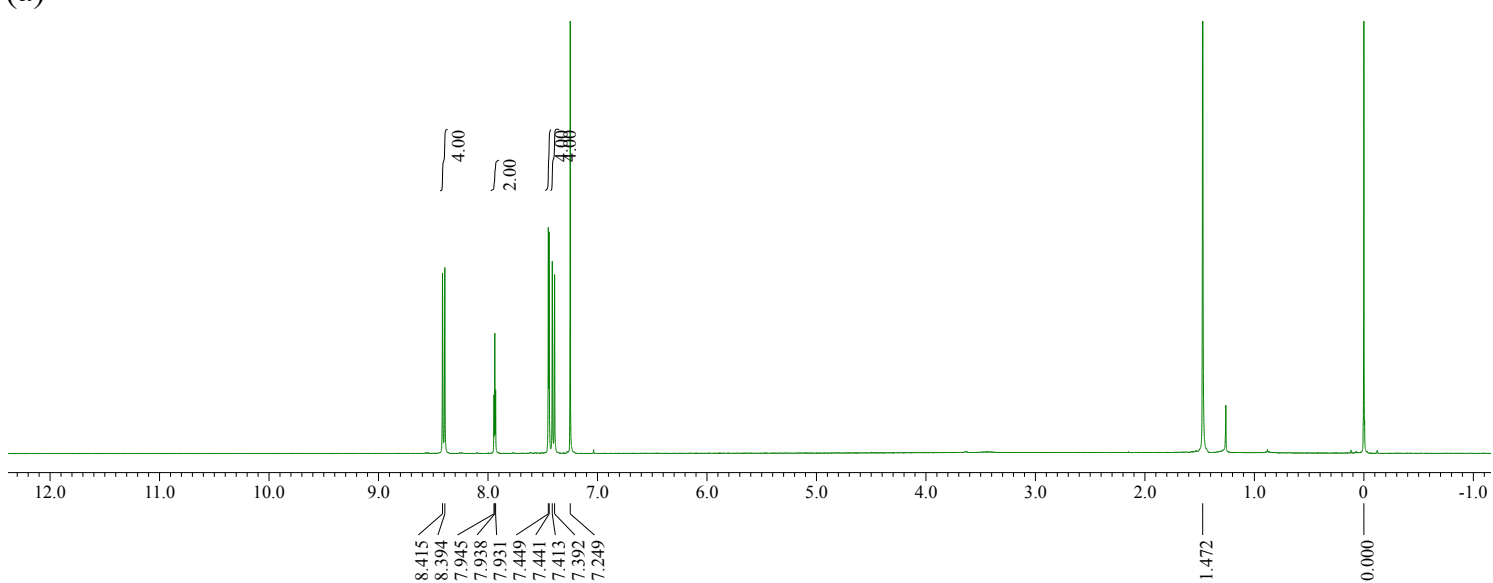

(b)

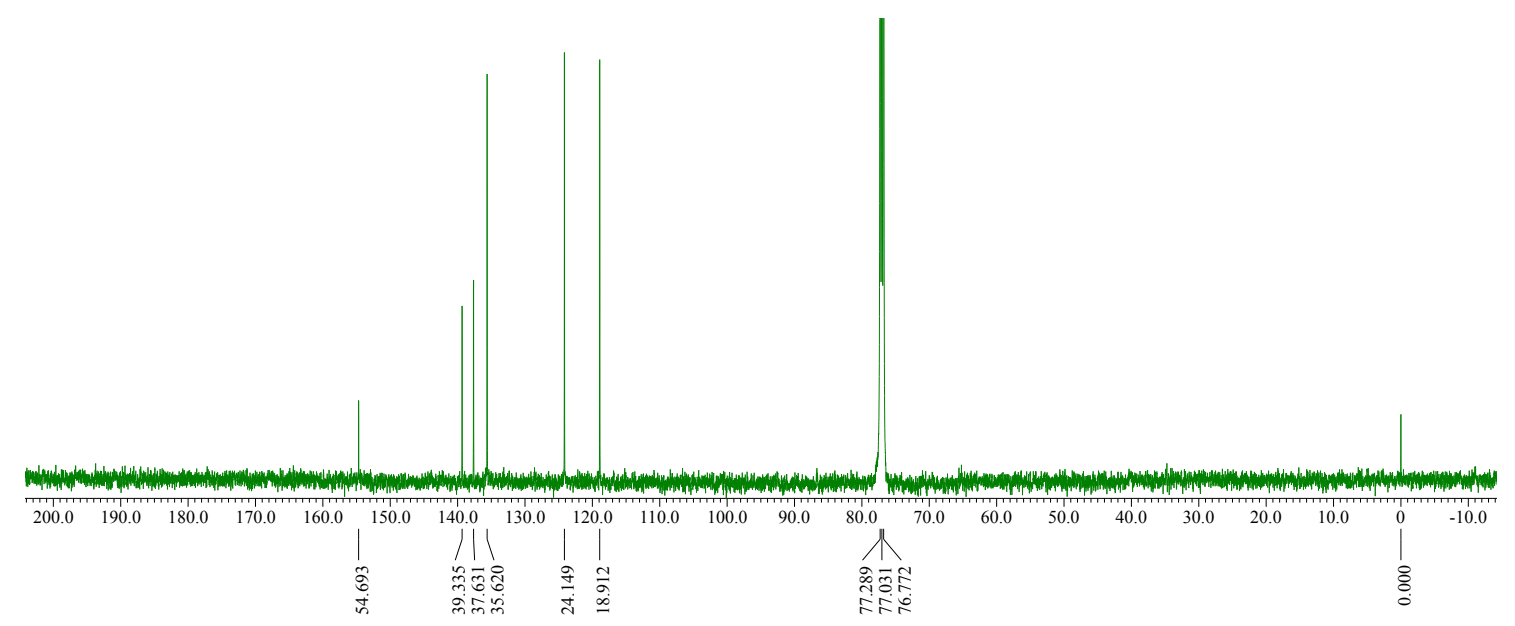

(c)

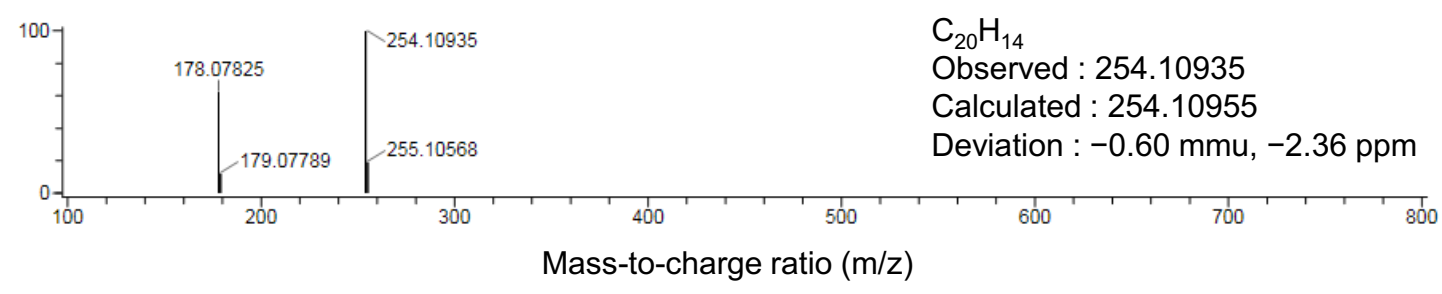

Figure S3. (a) ${ }^{1} \mathrm{H}$ NMR spectrum $\left(500 \mathrm{MHz}, \mathrm{CDCl}_{3}, 50{ }^{\circ} \mathrm{C}\right)$, (b) ${ }^{13} \mathrm{C}$ NMR spectrum $\left(125 \mathrm{MHz}, \mathrm{CDCl}_{3}\right.$, $50{ }^{\circ} \mathrm{C}$ ), and (c) FD-HRMS spectrum of BAz3. Anthracene [calcd for $\mathrm{C}_{14} \mathrm{H}_{10}\left(M^{+}\right): m / z=178.07825$ ] was used as an internal standard in HRMS analysis. 


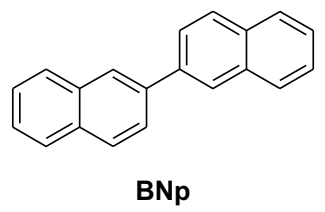

(a)

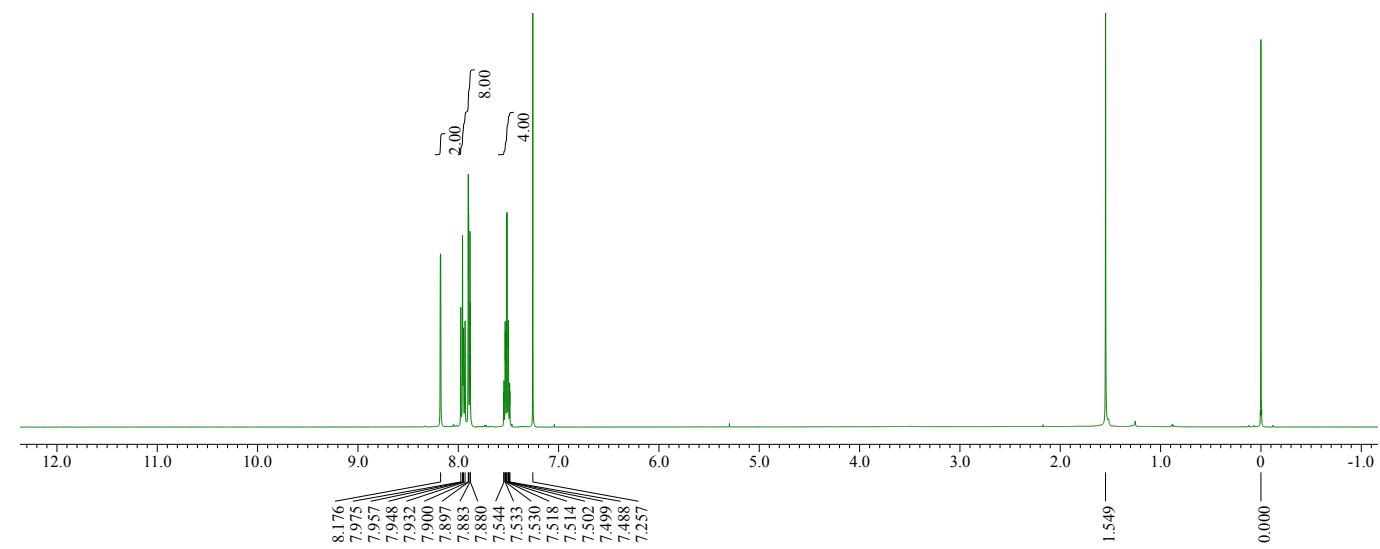

(b)

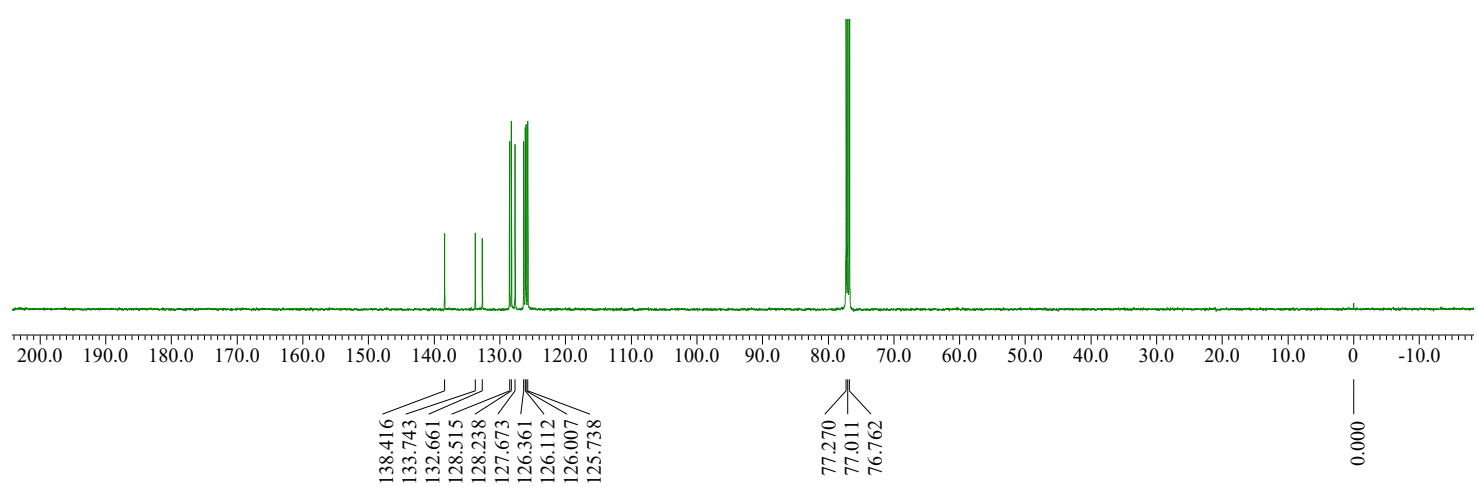

(c)

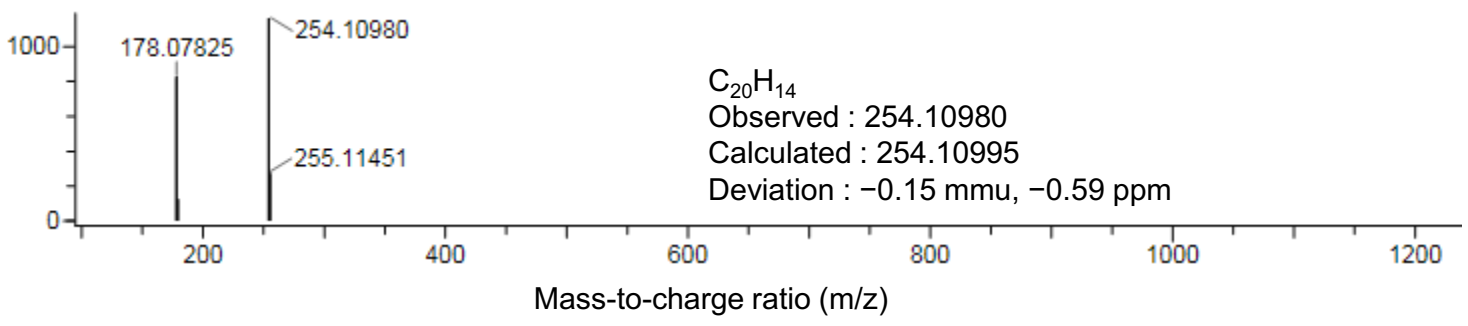

Figure S4. (a) ${ }^{1} \mathrm{H}$ NMR spectrum $\left(500 \mathrm{MHz}, \mathrm{CDCl}_{3}, 25^{\circ} \mathrm{C}\right)$, (b) ${ }^{13} \mathrm{C} \mathrm{NMR}$ spectrum $\left(125 \mathrm{MHz}, \mathrm{CDCl}_{3}\right.$, $25^{\circ} \mathrm{C}$ ), and (c) FD-HRMS spectrum of BNp. Anthracene [calcd for $\mathrm{C}_{14} \mathrm{H}_{10}\left(M^{+}\right): m / z=178.07825$ ] was used as an internal standard in HRMS analysis. 


\section{Cartesian coordinates and energies of all optimized structures}

Table S1. Cartesian coordinates of the optimized geometry of azulene computed at B3LYP/6$31+\mathrm{G}(\mathrm{d}, \mathrm{p})$ level of theory $(\mathrm{E}=-385.865765957$, number of imaginary frequencies $=0)$.

\begin{tabular}{llll}
\hline Atom & $\mathrm{X}$ & $\mathrm{Y}$ & $\mathrm{Z}$ \\
\hline $\mathrm{C}$ & 0.2708429 & 0.1427165 & -0.0043041 \\
$\mathrm{C}$ & 1.6704616 & 0.1389483 & -0.0043223 \\
$\mathrm{C}$ & 2.5478728 & 1.2294910 & -0.0037647 \\
$\mathrm{C}$ & -0.6224318 & 1.2202239 & -0.0037318 \\
$\mathrm{C}$ & 2.2550728 & 2.5918335 & -0.0030510 \\
$\mathrm{C}$ & -0.3391311 & 2.5909418 & -0.0030193 \\
$\mathrm{C}$ & 0.8982918 & 3.2316629 & -0.0026994 \\
$\mathrm{H}$ & -0.1934446 & -0.8418303 & -0.0048115 \\
$\mathrm{H}$ & 2.1387440 & -0.8423370 & -0.0048392 \\
$\mathrm{H}$ & 3.6088782 & 0.9815853 & -0.0039078 \\
$\mathrm{H}$ & -1.6774580 & 0.9572974 & -0.0038567 \\
$\mathrm{H}$ & -1.2053370 & 3.2519048 & -0.0026643 \\
$\mathrm{C}$ & 3.1933452 & 3.6414844 & -0.0025176 \\
$\mathrm{H}$ & 4.2693294 & 3.5171747 & -0.0025946 \\
$\mathrm{C}$ & 2.4967564 & 4.8628207 & -0.0018708 \\
$\mathrm{H}$ & 2.9592724 & 5.8436045 & -0.0013641 \\
$\mathrm{C}$ & 1.1112787 & 4.6233365 & -0.0019838 \\
$\mathrm{H}$ & 0.3308621 & 5.3744344 & -0.0015879 \\
\hline
\end{tabular}

Table S2. Cartesian coordinates of the optimized geometry of BAz1 computed at B3LYP/6-31+G(d,p) level of theory $(E=-770.535982771$, number of imaginary frequencies $=0)$.

\begin{tabular}{llll}
\hline Atom & $\mathrm{X}$ & $\mathrm{Y}$ & $\mathrm{Z}$ \\
\hline $\mathrm{C}$ & -4.0402341 & 0.114377 & -0.1208091 \\
$\mathrm{C}$ & -4.1342495 & 0.7949143 & 1.0992224 \\
$\mathrm{C}$ & -3.6713909 & 2.073362 & 1.4257215 \\
$\mathrm{C}$ & -3.4717554 & 0.5235591 & -1.3331869 \\
$\mathrm{C}$ & -3.0029713 & 2.9966602 & 0.6199467 \\
$\mathrm{C}$ & -2.8496797 & 1.7371363 & -1.6421828 \\
$\mathrm{C}$ & -2.6334374 & 2.8458861 & -0.8221603 \\
$\mathrm{H}$ & -4.4746886 & -0.8834794 & -0.1278192 \\
$\mathrm{H}$ & -4.629989 & 0.2546537 & 1.9020425
\end{tabular}




\begin{tabular}{llll}
$\mathrm{H}$ & -3.8522291 & 2.3945824 & 2.4508406 \\
$\mathrm{H}$ & -3.5253132 & -0.1978841 & -2.1448572 \\
$\mathrm{H}$ & -2.4860778 & 1.8357066 & -2.6643519 \\
$\mathrm{C}$ & -2.5575201 & 4.2658871 & 1.0174649 \\
$\mathrm{H}$ & -2.7055842 & 4.6926135 & 2.001445 \\
$\mathrm{C}$ & -1.951132 & 4.9129307 & -0.0871541 \\
$\mathrm{C}$ & -2.0058897 & 4.0412782 & -1.2023933 \\
$\mathrm{H}$ & -1.5876032 & 4.2352571 & -2.1820076 \\
$\mathrm{C}$ & -1.3610721 & 6.2680705 & -0.077758 \\
$\mathrm{C}$ & -0.7884067 & 6.703875 & 1.1371689 \\
$\mathrm{C}$ & -1.4259367 & 6.9981368 & -1.2847083 \\
$\mathrm{C}$ & -0.168233 & 7.9129295 & 1.4555587 \\
$\mathrm{H}$ & -0.8140179 & 5.9784911 & 1.944707 \\
$\mathrm{C}$ & -0.9614692 & 8.2792291 & -1.5861453 \\
$\mathrm{H}$ & -1.9355311 & 6.4946224 & -2.1006419 \\
$\mathrm{C}$ & 0.0670341 & 9.0337278 & 0.659875 \\
$\mathrm{H}$ & 0.1944036 & 7.9896887 & 2.4803218 \\
$\mathrm{C}$ & -0.3049556 & 9.2055857 & -0.7765094 \\
$\mathrm{H}$ & -1.1471487 & 8.6087623 & -2.6081374 \\
$\mathrm{C}$ & 0.7069364 & 10.2225545 & 1.0571987 \\
$\mathrm{C}$ & 0.1317087 & 10.488251 & -1.1569491 \\
$\mathrm{H}$ & 1.1015113 & 10.4211303 & 2.0462129 \\
$\mathrm{C}$ & 0.7422019 & 11.0966362 & -0.0448047 \\
$\mathrm{H}$ & 0.0133141 & 10.9237695 & -2.1416204 \\
$\mathrm{H}$ & 1.1752644 & 12.0907718 & -0.0380155 \\
\hline & & & \\
\hline & & & \\
$\mathrm{H}$ & & & \\
$\mathrm{H}$ & & &
\end{tabular}

Table S3. Cartesian coordinates of the optimized geometry of BAz2 computed at B3LYP/6-31+G(d,p) level of theory $(E=-770.542511076$, number of imaginary frequencies $=0)$.

\begin{tabular}{llll}
\hline Atom & $\mathrm{X}$ & $\mathrm{Y}$ & $\mathrm{Z}$ \\
\hline $\mathrm{C}$ & -2.5653114 & -0.3359831 & 0.2117555 \\
$\mathrm{C}$ & -2.3258626 & 0.2087194 & -1.0559547 \\
$\mathrm{C}$ & -1.7771488 & 1.4517289 & -1.3854199 \\
$\mathrm{C}$ & -2.3239367 & 0.2077425 & 1.479521 \\
$\mathrm{C}$ & -1.3281246 & 2.4666301 & -0.5373647 \\
$\mathrm{C}$ & -1.7747046 & 1.4504913 & 1.8090968 \\
$\mathrm{C}$ & -1.3269764 & 2.4660413 & 0.9611361
\end{tabular}




\begin{tabular}{llll}
$\mathrm{H}$ & -3.0050731 & -1.3315739 & 0.2117049 \\
$\mathrm{H}$ & -2.6045048 & -0.4204021 & -1.8979675 \\
$\mathrm{H}$ & -1.6856151 & 1.6612043 & -2.4505906 \\
$\mathrm{H}$ & -2.6012917 & -0.4220258 & 2.3214746 \\
$\mathrm{H}$ & -1.681525 & 1.6591474 & 2.874286 \\
$\mathrm{C}$ & -0.786223 & 3.694633 & -0.9379094 \\
$\mathrm{H}$ & -0.6499827 & 4.0054677 & -1.9660528 \\
$\mathrm{C}$ & -0.4502426 & 4.4530735 & 0.2119952 \\
$\mathrm{C}$ & -0.7844471 & 3.6937282 & 1.3618148 \\
$\mathrm{H}$ & -0.6466212 & 4.0037421 & 2.3899947 \\
$\mathrm{C}$ & 0.1368256 & 5.7826664 & 0.212066 \\
$\mathrm{C}$ & 0.471010 & 6.5420221 & -0.937758 \\
$\mathrm{C}$ & 0.4728252 & 6.5410968 & 1.3619661 \\
$\mathrm{C}$ & 1.0135382 & 7.7697057 & -0.5370831 \\
$\mathrm{H}$ & 0.3331646 & 6.2320056 & -1.9659346 \\
$\mathrm{C}$ & 1.0147269 & 7.7691035 & 0.9614177 \\
$\mathrm{H}$ & 0.3366042 & 6.2302648 & 2.3901129 \\
$\mathrm{C}$ & 1.4612449 & 8.7852781 & -1.3850316 \\
$\mathrm{C}$ & 1.4637728 & 8.7839822 & 1.809485 \\
$\mathrm{C}$ & 2.010474 & 10.0280254 & -1.0554357 \\
$\mathrm{H}$ & 1.3680525 & 8.576647 & -2.4502242 \\
$\mathrm{C}$ & 2.0124901 & 10.0269929 & 1.4800399 \\
$\mathrm{H}$ & 1.3722513 & 8.574482 & 2.8746523 \\
$\mathrm{C}$ & 2.251894 & 10.5717231 & 0.212332 \\
$\mathrm{H}$ & 2.2878084 & 10.6578134 & -1.897382 \\
$\mathrm{H}$ & 2.291153 & 10.6560947 & 2.3220599 \\
$\mathrm{H}$ & 2.6916559 & 11.5673138 & 0.2123869 \\
\hline & & & \\
\hline & & & \\
$\mathrm{H}$ & & & \\
$\mathrm{H}$ & & & \\
$\mathrm{H}$ & & & \\
$\mathrm{H}$ & & &
\end{tabular}

Table S4. Cartesian coordinates of the optimized geometry of BAz3 computed at B3LYP/6-31+G(d,p) level of theory $(E=-770.529177528$, number of imaginary frequencies $=0)$.

\begin{tabular}{llll}
\hline Atom & $\mathrm{X}$ & $\mathrm{Y}$ & $\mathrm{Z}$ \\
\hline $\mathrm{C}$ & -7.6491969 & -5.1587211 & 0.0452157 \\
$\mathrm{C}$ & -6.871469 & -4.8411197 & 1.1761877 \\
$\mathrm{C}$ & -6.1405079 & -3.684959 & 1.4649508 \\
$\mathrm{C}$ & -7.8726676 & -4.3479799 & -1.0850976 \\
$\mathrm{C}$ & -5.9824324 & -2.5208338 & 0.716458
\end{tabular}




\begin{tabular}{|c|c|c|c|}
\hline $\mathrm{C}$ & -7.4040172 & -3.0626193 & -1.3725833 \\
\hline $\mathrm{C}$ & -6.5792661 & -2.2268515 & -0.6230837 \\
\hline $\mathrm{H}$ & -6.8105215 & -5.6214493 & 1.9296682 \\
\hline $\mathrm{H}$ & -5.6097731 & -3.6999769 & 2.416459 \\
\hline $\mathrm{H}$ & -8.5273227 & -4.7759168 & -1.839199 \\
\hline $\mathrm{H}$ & -7.7399253 & -2.6508207 & -2.3238258 \\
\hline $\mathrm{C}$ & -5.225862 & -1.390534 & 1.0781341 \\
\hline $\mathrm{H}$ & -4.6631858 & -1.2816643 & 1.9971871 \\
\hline $\mathrm{C}$ & -5.3299693 & -0.4386668 & 0.0477046 \\
\hline $\mathrm{H}$ & -4.8517883 & 0.534520 & 0.0482226 \\
\hline $\mathrm{C}$ & -6.1466723 & -0.9369934 & -0.9834859 \\
\hline $\mathrm{H}$ & -6.4039288 & -0.4242813 & -1.9022137 \\
\hline $\mathrm{C}$ & -8.3128311 & -6.5096676 & 0.0444302 \\
\hline $\mathrm{C}$ & -9.0905006 & -6.8286126 & 1.1750646 \\
\hline $\mathrm{C}$ & -8.0894191 & -7.3190655 & -1.0868563 \\
\hline $\mathrm{C}$ & -9.8214497 & -7.9851143 & 1.4624899 \\
\hline $\mathrm{H}$ & -9.1514077 & -6.0491799 & 1.9294762 \\
\hline $\mathrm{C}$ & -8.5580889 & -8.6040815 & -1.3758474 \\
\hline $\mathrm{H}$ & -7.4348003 & -6.8902346 & -1.8404811 \\
\hline $\mathrm{C}$ & -9.9795688 & -9.1483472 & 0.7126197 \\
\hline $\mathrm{H}$ & -10.352135 & -7.9712275 & 2.414043 \\
\hline $\mathrm{C}$ & -9.3828062 & -9.440737 & -0.6273016 \\
\hline $\mathrm{H}$ & -8.2222304 & -9.0147495 & -2.3275959 \\
\hline $\mathrm{C}$ & -10.736125 & -10.279073 & 1.0729899 \\
\hline $\mathrm{C}$ & -9.8154246 & -10.730163 & -0.9892166 \\
\hline $\mathrm{H}$ & -11.298753 & -10.389034 & 1.9919425 \\
\hline $\mathrm{C}$ & -10.632076 & -11.229714 & 0.0414226 \\
\hline $\mathrm{H}$ & -9.5582184 & -11.241782 & -1.9085678 \\
\hline $\mathrm{H}$ & -11.110261 & -12.202899 & 0.0408073 \\
\hline
\end{tabular}

Table S5. Cartesian coordinates of the optimized geometry of BNp (transoid) computed at B3LYP/6$31+G(d, p)$ level of theory $(E=-770.641301809$, number of imaginary frequencies $=0)$.

\begin{tabular}{llll}
\hline Atom & $\mathrm{X}$ & $\mathrm{Y}$ & $\mathrm{Z}$ \\
\hline $\mathrm{C}$ & -6.3045049 & -1.5017438 & -2.9842181 \\
$\mathrm{C}$ & -4.9262725 & -1.4821121 & -2.9830473 \\
$\mathrm{C}$ & -4.1809436 & -2.6248939 & -2.5780906
\end{tabular}




\begin{tabular}{|c|c|c|c|}
\hline $\mathrm{C}$ & -4.8897729 & -3.8034068 & -2.175168 \\
\hline $\mathrm{C}$ & -6.310894 & -3.791638 & -2.1856379 \\
\hline $\mathrm{C}$ & -7.0044785 & -2.667371 & -2.5808402 \\
\hline $\mathrm{H}$ & -2.2383982 & -1.7420752 & -2.9001659 \\
\hline $\mathrm{H}$ & -6.8607134 & -0.6219984 & -3.2947721 \\
\hline $\mathrm{H}$ & -4.3892687 & -0.5888935 & -3.2922373 \\
\hline $\mathrm{C}$ & -2.7609803 & -2.6349468 & -2.5671127 \\
\hline $\mathrm{C}$ & -4.1355198 & -4.9410689 & -1.776810 \\
\hline $\mathrm{H}$ & -6.8447767 & -4.6868638 & -1.8767382 \\
\hline $\mathrm{H}$ & -8.0905694 & -2.6708478 & -2.585625 \\
\hline $\mathrm{C}$ & -2.760103 & -4.9182992 & -1.7785343 \\
\hline $\mathrm{C}$ & -2.0392682 & -3.7531704 & -2.1792621 \\
\hline $\mathrm{H}$ & -4.6642628 & -5.8352868 & -1.4562762 \\
\hline $\mathrm{H}$ & -2.2079495 & -5.791254 & -1.4442268 \\
\hline $\mathrm{C}$ & -0.5536832 & -3.7507184 & -2.1822631 \\
\hline $\mathrm{C}$ & 0.1664681 & -4.8690512 & -2.5726902 \\
\hline $\mathrm{C}$ & 0.1687522 & -2.5854611 & -1.7848038 \\
\hline $\mathrm{C}$ & 1.5863769 & -4.8790894 & -2.589409 \\
\hline $\mathrm{H}$ & -0.3574457 & -5.7620304 & -2.9033543 \\
\hline $\mathrm{C}$ & 1.5441626 & -2.5626712 & -1.7886585 \\
\hline $\mathrm{H}$ & -0.3820545 & -1.7124126 & -1.4485264 \\
\hline $\mathrm{C}$ & 2.2968132 & -3.7004408 & -2.1897277 \\
\hline $\mathrm{C}$ & 2.3300799 & -6.0219831 & -2.9970358 \\
\hline $\mathrm{H}$ & 2.0741851 & -1.6683479 & -1.4705412 \\
\hline $\mathrm{C}$ & 3.7178805 & -3.712188 & -2.2059527 \\
\hline $\mathrm{C}$ & 3.7082973 & -6.0023258 & -3.003805 \\
\hline $\mathrm{H}$ & 1.7918421 & -6.9153049 & -3.3037723 \\
\hline $\mathrm{C}$ & 4.4098793 & -4.8365622 & -2.6036259 \\
\hline $\mathrm{H}$ & 4.2529926 & -2.8168579 & -1.8994921 \\
\hline $\mathrm{H}$ & 4.2632607 & -6.8821566 & -3.316339 \\
\hline $\mathrm{H}$ & 5.4959419 & -4.8330716 & -2.6128105 \\
\hline
\end{tabular}

Table S6. Cartesian coordinates of the optimized geometry of BNp (cisoid) computed at B3LYP/6$31+\mathrm{G}(\mathrm{d}, \mathrm{p})$ level of theory $(\mathrm{E}=-770.641276065$, number of imaginary frequencies $=0)$.

\begin{tabular}{l|l|l|l}
\hline Atom & $\mathrm{X}$ & $\mathrm{Y}$ & $\mathrm{Z}$ \\
\hline $\mathrm{C}$ & -6.331151 & -3.156750 & -0.133815
\end{tabular}




\begin{tabular}{|c|c|c|c|}
\hline $\mathrm{C}$ & -4.956189 & -3.061298 & -0.121004 \\
\hline $\mathrm{C}$ & -4.188381 & -3.437073 & -1.258898 \\
\hline $\mathrm{C}$ & -4.871486 & -3.912323 & -2.425782 \\
\hline $\mathrm{C}$ & -6.290012 & -3.999603 & -2.407463 \\
\hline $\mathrm{C}$ & -7.005396 & -3.631390 & -1.287785 \\
\hline $\mathrm{H}$ & -2.267043 & -2.959376 & -0.395490 \\
\hline $\mathrm{H}$ & -6.904617 & -2.867959 & 0.742259 \\
\hline $\mathrm{H}$ & -4.438841 & -2.697114 & 0.763028 \\
\hline $\mathrm{C}$ & -2.771307 & -3.348224 & -1.276282 \\
\hline $\mathrm{C}$ & -4.096002 & -4.277510 & -3.560192 \\
\hline $\mathrm{H}$ & -6.804318 & -4.362450 & -3.293868 \\
\hline $\mathrm{H}$ & -8.089181 & -3.702392 & -1.285973 \\
\hline $\mathrm{C}$ & -2.723761 & -4.178764 & -3.544786 \\
\hline $\mathrm{C}$ & -2.028859 & -3.711971 & -2.388711 \\
\hline $\mathrm{H}$ & -4.604900 & -4.647882 & -4.446628 \\
\hline $\mathrm{H}$ & -2.153679 & -4.485730 & -4.416476 \\
\hline $\mathrm{C}$ & -0.545943 & -3.622350 & -2.389633 \\
\hline $\mathrm{C}$ & 0.197929 & -3.986788 & -1.278380 \\
\hline $\mathrm{C}$ & 0.147480 & -3.154864 & -3.546314 \\
\hline $\mathrm{C}$ & 1.615024 & -3.897974 & -1.262763 \\
\hline $\mathrm{H}$ & -0.305211 & -4.376167 & -0.397181 \\
\hline $\mathrm{C}$ & 1.519704 & -3.056132 & -3.563424 \\
\hline $\mathrm{H}$ & -0.423715 & -2.847357 & -4.417084 \\
\hline $\mathrm{C}$ & 2.296638 & -3.422026 & -2.430236 \\
\hline $\mathrm{C}$ & 2.384289 & -4.274457 & -0.126085 \\
\hline $\mathrm{H}$ & 2.027467 & -2.685229 & -4.450288 \\
\hline $\mathrm{C}$ & 3.715190 & -3.334790 & -2.413691 \\
\hline $\mathrm{C}$ & 3.759235 & -4.179023 & -0.140607 \\
\hline $\mathrm{H}$ & 1.868069 & -4.639167 & 0.758389 \\
\hline $\mathrm{C}$ & 4.432007 & -3.703697 & -1.295158 \\
\hline $\mathrm{H}$ & 4.228361 & -2.971422 & -3.300539 \\
\hline $\mathrm{H}$ & 4.333823 & -4.468357 & 0.734553 \\
\hline $\mathrm{H}$ & 5.515796 & -3.632726 & -1.294697 \\
\hline
\end{tabular}

Table S7. Cartesian coordinates of the optimized geometry of BAzl computed at CAM-B3LYP/6$31+\mathrm{G}(\mathrm{d}, \mathrm{p})$ level of theory (charge $=0$; spin multiplicity $=1, \mathrm{E}=-770.063804863$, number of 
imaginary frequencies $=0$ ).

\begin{tabular}{|c|c|c|c|}
\hline Atom & $\mathrm{X}$ & $\mathrm{Y}$ & Z \\
\hline $\mathrm{C}$ & -5.854993 & -0.000001 & -0.000003 \\
\hline $\mathrm{C}$ & -5.264337 & -1.202424 & 0.386238 \\
\hline $\mathrm{C}$ & -3.911299 & -1.516080 & 0.479809 \\
\hline $\mathrm{C}$ & -5.264335 & 1.202426 & -0.386238 \\
\hline $\mathrm{C}$ & -2.807525 & -0.712474 & 0.216604 \\
\hline $\mathrm{C}$ & -3.911297 & 1.516085 & -0.479802 \\
\hline $\mathrm{C}$ & -2.807524 & 0.712475 & -0.216599 \\
\hline $\mathrm{H}$ & -6.942415 & -0.000003 & -0.000012 \\
\hline $\mathrm{H}$ & -5.952255 & -2.002394 & 0.644579 \\
\hline $\mathrm{H}$ & -3.681177 & -2.530812 & 0.799287 \\
\hline $\mathrm{H}$ & -5.952254 & 2.002395 & -0.644581 \\
\hline $\mathrm{H}$ & -3.681175 & 2.530820 & -0.799268 \\
\hline $\mathrm{C}$ & -1.467879 & -1.097631 & 0.325148 \\
\hline $\mathrm{H}$ & -1.127022 & -2.071158 & 0.652815 \\
\hline $\mathrm{C}$ & -0.648502 & -0.000002 & -0.000002 \\
\hline $\mathrm{C}$ & -1.467879 & 1.097627 & -0.325152 \\
\hline $\mathrm{H}$ & -1.127023 & 2.071154 & -0.652823 \\
\hline $\mathrm{C}$ & 0.830395 & -0.000002 & -0.000002 \\
\hline $\mathrm{C}$ & 1.458403 & -1.196801 & -0.380396 \\
\hline $\mathrm{C}$ & 1.458403 & 1.196799 & 0.380392 \\
\hline $\mathrm{C}$ & 2.810843 & -1.503863 & -0.473216 \\
\hline $\mathrm{H}$ & 0.785350 & -1.999369 & -0.663909 \\
\hline $\mathrm{C}$ & 2.810841 & 1.503862 & 0.473214 \\
\hline $\mathrm{H}$ & 0.785349 & 1.999367 & 0.663903 \\
\hline $\mathrm{C}$ & 3.922742 & -0.709527 & -0.221599 \\
\hline $\mathrm{H}$ & 3.033900 & -2.518284 & -0.799722 \\
\hline $\mathrm{C}$ & 3.922741 & 0.709527 & 0.221599 \\
\hline $\mathrm{H}$ & 3.033896 & 2.518285 & 0.799717 \\
\hline $\mathrm{C}$ & 5.265282 & -1.094485 & -0.342734 \\
\hline $\mathrm{C}$ & 5.265280 & 1.094486 & 0.342741 \\
\hline $\mathrm{H}$ & 5.610041 & -2.073679 & -0.649631 \\
\hline $\mathrm{C}$ & 6.069263 & 0.000003 & -0.000003 \\
\hline $\mathrm{H}$ & 5.610036 & 2.073679 & 0.649643 \\
\hline $\mathrm{H}$ & 7.153021 & 0.000003 & 0.000000 \\
\hline
\end{tabular}


Table S8. Cartesian coordinates of the optimized geometry of BAzl cation computed at CAM$\mathrm{B} 3 \mathrm{LYP} / 6-31+\mathrm{G}(\mathrm{d}, \mathrm{p})$ level of theory $($ charge $=+1$; spin multiplicity $=2, \mathrm{E}=-769.816495018$, number of imaginary frequencies $=0$ ).

\begin{tabular}{|c|c|c|c|}
\hline Atom & $X$ & $\mathrm{Y}$ & Z \\
\hline $\mathrm{C}$ & -5.852676 & -0.000006 & -0.000003 \\
\hline $\mathrm{C}$ & -5.265304 & -1.232835 & 0.288373 \\
\hline $\mathrm{C}$ & -3.916075 & -1.551896 & 0.355310 \\
\hline $\mathrm{C}$ & -5.265310 & 1.232824 & -0.288381 \\
\hline $\mathrm{C}$ & -2.810982 & -0.723014 & 0.154598 \\
\hline $\mathrm{C}$ & -3.916083 & 1.551893 & -0.355315 \\
\hline $\mathrm{C}$ & -2.810985 & 0.723019 & -0.154589 \\
\hline $\mathrm{H}$ & -6.939715 & -0.000008 & 0.000005 \\
\hline $\mathrm{H}$ & -5.955097 & -2.048508 & 0.481145 \\
\hline $\mathrm{H}$ & -3.685013 & -2.588459 & 0.591756 \\
\hline $\mathrm{H}$ & -5.955106 & 2.048492 & -0.481164 \\
\hline $\mathrm{H}$ & -3.685029 & 2.588450 & -0.591795 \\
\hline $\mathrm{C}$ & -1.475249 & -1.126949 & 0.226942 \\
\hline $\mathrm{H}$ & -1.150725 & -2.127041 & 0.482048 \\
\hline $\mathrm{C}$ & -0.655113 & 0.000005 & 0.000003 \\
\hline $\mathrm{C}$ & -1.475255 & 1.126960 & -0.226931 \\
\hline $\mathrm{H}$ & -1.150727 & 2.127051 & -0.482030 \\
\hline $\mathrm{C}$ & 0.805133 & 0.000009 & 0.000003 \\
\hline $\mathrm{C}$ & 1.457508 & -1.219583 & -0.287184 \\
\hline $\mathrm{C}$ & 1.457509 & 1.219594 & 0.287192 \\
\hline $\mathrm{C}$ & 2.809475 & -1.530342 & -0.348744 \\
\hline $\mathrm{H}$ & 0.793137 & -2.046556 & -0.512078 \\
\hline $\mathrm{C}$ & 2.809481 & 1.530342 & 0.348749 \\
\hline $\mathrm{H}$ & 0.793148 & 2.046576 & 0.512082 \\
\hline $\mathrm{C}$ & 3.903143 & -0.694563 & -0.157093 \\
\hline $\mathrm{H}$ & 3.039748 & -2.564795 & -0.591592 \\
\hline $\mathrm{C}$ & 3.903145 & 0.694559 & 0.157091 \\
\hline $\mathrm{H}$ & 3.039759 & 2.564791 & 0.591609 \\
\hline $\mathrm{C}$ & 5.295326 & -1.101270 & -0.250537 \\
\hline $\mathrm{C}$ & 5.295324 & 1.101262 & 0.250531 \\
\hline $\mathrm{H}$ & 5.625848 & -2.107166 & -0.479461 \\
\hline $\mathrm{C}$ & 6.109214 & -0.000005 & -0.000010 \\
\hline
\end{tabular}




\begin{tabular}{llll}
$\mathrm{H}$ & 5.625855 & 2.107153 & 0.479459 \\
$\mathrm{H}$ & 7.190569 & 0.000000 & -0.000013 \\
\hline
\end{tabular}

Table S9. Cartesian coordinates of the optimized geometry of BAz2 computed at CAM-B3LYP/6$31+\mathrm{G}(\mathrm{d}, \mathrm{p})$ level of theory (charge $=0$; spin multiplicity $=1, \mathrm{E}=-770.069054082$, number of imaginary frequencies $=0$ ).

\begin{tabular}{|c|c|c|c|}
\hline Atom & $X$ & $\mathrm{Y}$ & Z \\
\hline $\mathrm{C}$ & 5.937278 & -0.000004 & 0.000004 \\
\hline $\mathrm{C}$ & 5.345901 & -1.262780 & -0.000011 \\
\hline $\mathrm{C}$ & 3.992967 & -1.590515 & -0.000017 \\
\hline $\mathrm{C}$ & 5.345902 & 1.262777 & 0.000017 \\
\hline $\mathrm{C}$ & 2.887824 & -0.745881 & -0.000008 \\
\hline $\mathrm{C}$ & 3.992973 & 1.590515 & 0.000015 \\
\hline $\mathrm{C}$ & 2.887824 & 0.745883 & 0.000004 \\
\hline $\mathrm{H}$ & 7.024734 & -0.000004 & 0.000009 \\
\hline $\mathrm{H}$ & 6.033802 & -2.103607 & -0.000022 \\
\hline $\mathrm{H}$ & 3.763289 & -2.654496 & -0.000027 \\
\hline $\mathrm{H}$ & 6.033807 & 2.103601 & 0.000030 \\
\hline $\mathrm{H}$ & 3.763297 & 2.654497 & 0.000024 \\
\hline $\mathrm{C}$ & 1.549839 & -1.144885 & -0.000012 \\
\hline $\mathrm{H}$ & 1.209990 & -2.172307 & -0.000022 \\
\hline $\mathrm{C}$ & 0.728423 & 0.000001 & -0.000003 \\
\hline $\mathrm{C}$ & 1.549843 & 1.144889 & 0.000006 \\
\hline $\mathrm{H}$ & 1.209993 & 2.172310 & 0.000015 \\
\hline $\mathrm{C}$ & -0.728424 & 0.000002 & -0.000002 \\
\hline $\mathrm{C}$ & -1.549842 & -1.144886 & 0.000007 \\
\hline $\mathrm{C}$ & -1.549841 & 1.144888 & -0.000009 \\
\hline $\mathrm{C}$ & -2.887823 & -0.745881 & 0.000006 \\
\hline $\mathrm{H}$ & -1.209991 & -2.172307 & 0.000015 \\
\hline $\mathrm{C}$ & -2.887825 & 0.745882 & -0.000005 \\
\hline $\mathrm{H}$ & -1.209992 & 2.172310 & -0.000017 \\
\hline $\mathrm{C}$ & -3.992970 & -1.590516 & 0.000018 \\
\hline $\mathrm{C}$ & -3.992969 & 1.590515 & -0.000016 \\
\hline $\mathrm{C}$ & -5.345901 & -1.262779 & 0.000016 \\
\hline $\mathrm{H}$ & -3.763293 & -2.654497 & 0.000030 \\
\hline $\mathrm{C}$ & -5.345902 & 1.262777 & -0.000012 \\
\hline
\end{tabular}




\begin{tabular}{llll}
$\mathrm{H}$ & -3.763294 & 2.654496 & -0.000028 \\
$\mathrm{C}$ & -5.937278 & 0.000000 & 0.000002 \\
$\mathrm{H}$ & -6.033804 & -2.103605 & 0.000026 \\
$\mathrm{H}$ & -6.033805 & 2.103603 & -0.000022 \\
$\mathrm{H}$ & -7.024735 & 0.000000 & 0.000000 \\
\hline
\end{tabular}

Table S10. Cartesian coordinates of the optimized geometry of BAz2 cation computed at CAM$\mathrm{B} 3 \mathrm{LYP} / 6-31+\mathrm{G}(\mathrm{d}, \mathrm{p})$ level of theory $($ charge $=+1$; spin multiplicity $=2, \mathrm{E}=-769.816974582$, number of imaginary frequencies $=0$ ).

\begin{tabular}{|c|c|c|c|}
\hline Atom & $\mathrm{X}$ & $\mathrm{Y}$ & Z \\
\hline $\mathrm{C}$ & 5.963501 & -0.000019 & 0.000003 \\
\hline $\mathrm{C}$ & 5.362678 & -1.257377 & -0.000003 \\
\hline $\mathrm{C}$ & 4.002249 & -1.586666 & -0.000005 \\
\hline $\mathrm{C}$ & 5.362681 & 1.257357 & 0.000008 \\
\hline $\mathrm{C}$ & 2.922519 & -0.722209 & -0.000006 \\
\hline $\mathrm{C}$ & 4.002268 & 1.586665 & 0.000007 \\
\hline $\mathrm{C}$ & 2.922518 & 0.722215 & 0.000001 \\
\hline $\mathrm{H}$ & 7.049313 & -0.000019 & -0.000004 \\
\hline $\mathrm{H}$ & 6.045264 & -2.102299 & -0.000013 \\
\hline $\mathrm{H}$ & 3.768757 & -2.648055 & -0.000003 \\
\hline $\mathrm{H}$ & 6.045281 & 2.102268 & 0.000003 \\
\hline $\mathrm{H}$ & 3.768788 & 2.648056 & 0.000003 \\
\hline $\mathrm{C}$ & 1.539068 & -1.127756 & -0.000001 \\
\hline $\mathrm{H}$ & 1.211999 & -2.159961 & -0.000002 \\
\hline $\mathrm{C}$ & 0.702705 & 0.000024 & -0.000002 \\
\hline $\mathrm{C}$ & 1.539075 & 1.127776 & 0.000003 \\
\hline $\mathrm{H}$ & 1.212022 & 2.159985 & 0.000011 \\
\hline $\mathrm{C}$ & -0.746206 & 0.000017 & 0.000002 \\
\hline $\mathrm{C}$ & -1.559913 & -1.148958 & 0.000005 \\
\hline $\mathrm{C}$ & -1.559929 & 1.148983 & -0.000003 \\
\hline $\mathrm{C}$ & -2.898240 & -0.740278 & 0.000000 \\
\hline $\mathrm{H}$ & -1.229020 & -2.179306 & 0.000010 \\
\hline $\mathrm{C}$ & -2.898247 & 0.740288 & -0.000006 \\
\hline $\mathrm{H}$ & -1.229045 & 2.179334 & -0.000009 \\
\hline $\mathrm{C}$ & -4.002993 & -1.591112 & -0.000002 \\
\hline $\mathrm{C}$ & -4.003019 & 1.591107 & -0.000013 \\
\hline
\end{tabular}




\begin{tabular}{llll}
$\mathrm{C}$ & -5.353193 & -1.264998 & 0.000006 \\
$\mathrm{H}$ & -3.772549 & -2.654460 & -0.000007 \\
$\mathrm{C}$ & -5.353208 & 1.264972 & -0.000001 \\
$\mathrm{H}$ & -3.772590 & 2.654458 & -0.000024 \\
$\mathrm{C}$ & -5.941807 & -0.000020 & 0.000009 \\
$\mathrm{H}$ & -6.042217 & -2.103906 & 0.000007 \\
$\mathrm{H}$ & -6.042248 & 2.103867 & 0.000001 \\
$\mathrm{H}$ & -7.028798 & -0.000026 & 0.000019 \\
\hline
\end{tabular}

Table S11. Cartesian coordinates of the optimized geometry of BAz3 computed at CAM-B3LYP/6$31+\mathrm{G}(\mathrm{d}, \mathrm{p})$ level of theory $($ charge $=0$; spin multiplicity $=1, \mathrm{E}=-770.05776131$, number of imaginary frequencies $=0$ ).

\begin{tabular}{|c|c|c|c|}
\hline Atom & $X$ & $\mathrm{Y}$ & Z \\
\hline $\mathrm{C}$ & 0.751467 & -0.000007 & 0.000010 \\
\hline $\mathrm{C}$ & 1.372884 & -1.118435 & -0.571838 \\
\hline $\mathrm{C}$ & 2.727016 & -1.403719 & -0.721638 \\
\hline $\mathrm{C}$ & 1.372886 & 1.118426 & 0.571854 \\
\hline $\mathrm{C}$ & 3.836653 & -0.661926 & -0.340584 \\
\hline $\mathrm{C}$ & 2.727014 & 1.403715 & 0.721646 \\
\hline $\mathrm{C}$ & 3.836656 & 0.661930 & 0.340578 \\
\hline $\mathrm{H}$ & 0.697732 & -1.866146 & -0.976627 \\
\hline $\mathrm{H}$ & 2.949570 & -2.347565 & -1.216339 \\
\hline $\mathrm{H}$ & 0.697733 & 1.866135 & 0.976644 \\
\hline $\mathrm{H}$ & 2.949566 & 2.347559 & 1.216352 \\
\hline $\mathrm{C}$ & 5.179319 & -1.019423 & -0.525619 \\
\hline $\mathrm{H}$ & 5.524602 & -1.931428 & -0.995436 \\
\hline $\mathrm{C}$ & 5.982758 & 0.000000 & -0.000009 \\
\hline $\mathrm{H}$ & 7.066438 & 0.000002 & -0.000014 \\
\hline $\mathrm{C}$ & 5.179320 & 1.019431 & 0.525601 \\
\hline $\mathrm{H}$ & 5.524609 & 1.931436 & 0.995413 \\
\hline $\mathrm{C}$ & -0.751467 & -0.000003 & 0.000007 \\
\hline $\mathrm{C}$ & -1.372891 & -1.118429 & 0.571861 \\
\hline $\mathrm{C}$ & -1.372880 & 1.118423 & -0.571845 \\
\hline $\mathrm{C}$ & -2.727018 & -1.403717 & 0.721648 \\
\hline $\mathrm{H}$ & -0.697739 & -1.866129 & 0.976670 \\
\hline $\mathrm{C}$ & -2.727011 & 1.403717 & -0.721636 \\
\hline
\end{tabular}




\begin{tabular}{llll}
$\mathrm{H}$ & -0.697727 & 1.866129 & -0.97664 \\
$\mathrm{C}$ & -3.836657 & -0.661930 & 0.340574 \\
$\mathrm{H}$ & -2.949573 & -2.347561 & 1.216352 \\
$\mathrm{C}$ & -3.836650 & 0.661932 & -0.340574 \\
$\mathrm{H}$ & -2.949561 & 2.347566 & -1.216334 \\
$\mathrm{C}$ & -5.179323 & -1.019428 & 0.525594 \\
$\mathrm{C}$ & -5.179318 & 1.019434 & -0.525615 \\
$\mathrm{H}$ & -5.524614 & -1.931432 & 0.995406 \\
$\mathrm{C}$ & -5.982758 & 0.000005 & -0.000016 \\
$\mathrm{H}$ & -5.524600 & 1.931442 & -0.995426 \\
$\mathrm{H}$ & -7.066438 & 0.000005 & -0.000022 \\
\hline
\end{tabular}

Table S12. Cartesian coordinates of the optimized geometry of BAz3 cation computed at CAM$\mathrm{B} 3 \mathrm{LYP} / 6-31+\mathrm{G}(\mathrm{d}, \mathrm{p})$ level of theory $($ charge $=+1$; spin multiplicity $=2, \mathrm{E}=-769.800643178$, number of imaginary frequencies $=0$ ).

\begin{tabular}{llll}
\hline Atom & $\mathrm{X}$ & $\mathrm{Y}$ & $\mathrm{Z}$ \\
\hline $\mathrm{C}$ & 0.766784 & -0.000010 & 0.000004 \\
$\mathrm{C}$ & 1.376822 & -1.147844 & -0.525121 \\
$\mathrm{C}$ & 2.731910 & -1.439374 & -0.671907 \\
$\mathrm{C}$ & 1.376815 & 1.147828 & 0.525131 \\
$\mathrm{C}$ & 3.834575 & -0.679613 & -0.318763 \\
$\mathrm{C}$ & 2.731899 & 1.439370 & 0.671915 \\
$\mathrm{C}$ & 3.834571 & 0.679619 & 0.318762 \\
$\mathrm{H}$ & 0.704909 & -1.910017 & -0.909127 \\
$\mathrm{H}$ & 2.954272 & -2.399348 & -1.132615 \\
$\mathrm{H}$ & 0.704896 & 1.909997 & 0.909136 \\
$\mathrm{H}$ & 2.954253 & 2.399343 & 1.132626 \\
$\mathrm{C}$ & 5.174380 & -1.039186 & -0.488677 \\
$\mathrm{H}$ & 5.524256 & -1.965979 & -0.924054 \\
$\mathrm{C}$ & 5.976850 & 0.000009 & 0.000001 \\
$\mathrm{H}$ & 7.060067 & 0.000010 & 0.000003 \\
$\mathrm{C}$ & 5.174374 & 1.039202 & 0.488674 \\
$\mathrm{H}$ & 5.524245 & 1.965998 & 0.924051 \\
$\mathrm{C}$ & -0.727413 & -0.000011 & -0.000006 \\
$\mathrm{C}$ & -1.368253 & -1.126847 & 0.545324 \\
$\mathrm{C}$ & -1.368246 & 1.126830 & -0.545337 \\
& & &
\end{tabular}




\begin{tabular}{llll}
$\mathrm{C}$ & -2.725412 & -1.416987 & 0.682410 \\
$\mathrm{H}$ & -0.698252 & -1.884361 & 0.939749 \\
$\mathrm{C}$ & -2.725402 & 1.416979 & -0.682418 \\
$\mathrm{H}$ & -0.698240 & 1.884342 & -0.939756 \\
$\mathrm{C}$ & -3.813610 & -0.644668 & 0.310951 \\
$\mathrm{H}$ & -2.954298 & -2.370208 & 1.152566 \\
$\mathrm{C}$ & -3.813605 & 0.644671 & -0.310951 \\
$\mathrm{H}$ & -2.954284 & 2.370199 & -1.152576 \\
$\mathrm{C}$ & -5.205355 & -1.017225 & 0.493001 \\
$\mathrm{C}$ & -5.205345 & 1.017242 & -0.493000 \\
$\mathrm{H}$ & -5.537352 & -1.945158 & 0.942636 \\
$\mathrm{C}$ & -6.017667 & 0.000012 & 0.000004 \\
$\mathrm{H}$ & -5.537332 & 1.945179 & -0.942636 \\
$\mathrm{H}$ & -7.099184 & 0.000018 & 0.000008 \\
\hline
\end{tabular}

Table S13. Cartesian coordinates of the optimized geometry of BNp computed at CAM-B3LYP/6$31+\mathrm{G}(\mathrm{d}, \mathrm{p})$ level of theory (charge $=0$; spin multiplicity $=1, \mathrm{E}=-770.182862547$, number of imaginary frequencies $=0$ ).

\begin{tabular}{llll}
\hline Atom & $\mathrm{X}$ & $\mathrm{Y}$ & $\mathrm{Z}$ \\
\hline $\mathrm{C}$ & -5.339113 & 1.152219 & -0.624727 \\
$\mathrm{C}$ & -4.002313 & 1.454121 & -0.621346 \\
$\mathrm{C}$ & -3.041964 & 0.496137 & -0.201659 \\
$\mathrm{C}$ & -3.489437 & -0.787835 & 0.211783 \\
$\mathrm{C}$ & -4.879210 & -1.071245 & 0.199133 \\
$\mathrm{C}$ & -5.783695 & -0.125491 & -0.208844 \\
$\mathrm{H}$ & -1.324908 & 1.758370 & -0.529364 \\
$\mathrm{H}$ & -6.063499 & 1.893413 & -0.946908 \\
$\mathrm{H}$ & -3.658796 & 2.434040 & -0.940373 \\
$\mathrm{C}$ & -1.653312 & 0.780655 & -0.188449 \\
$\mathrm{C}$ & -2.521996 & -1.739589 & 0.625830 \\
$\mathrm{H}$ & -5.215801 & -2.053497 & 0.518471 \\
$\mathrm{H}$ & -6.844790 & -0.353129 & -0.215416 \\
$\mathrm{C}$ & -1.188196 & -1.434828 & 0.626647 \\
$\mathrm{C}$ & -0.725923 & -0.154177 & 0.212885 \\
$\mathrm{H}$ & -2.857724 & -2.718757 & 0.955393 \\
$\mathrm{H}$ & -0.467211 & -2.169119 & 0.970575 \\
& & &
\end{tabular}




\begin{tabular}{llll}
$\mathrm{C}$ & 0.725925 & 0.154158 & 0.212893 \\
$\mathrm{C}$ & 1.653321 & -0.780664 & -0.188449 \\
$\mathrm{C}$ & 1.188189 & 1.434810 & 0.626663 \\
$\mathrm{C}$ & 3.041969 & -0.496137 & -0.201657 \\
$\mathrm{H}$ & 1.324924 & -1.758377 & -0.529377 \\
$\mathrm{C}$ & 2.521985 & 1.739582 & 0.625835 \\
$\mathrm{H}$ & 0.467199 & 2.169097 & 0.970589 \\
$\mathrm{C}$ & 3.489433 & 0.787835 & 0.211787 \\
$\mathrm{C}$ & 4.002324 & -1.454116 & -0.621343 \\
$\mathrm{H}$ & 2.857705 & 2.718757 & 0.955385 \\
$\mathrm{C}$ & 4.879201 & 1.071254 & 0.199133 \\
$\mathrm{C}$ & 5.339121 & -1.152202 & -0.624732 \\
$\mathrm{H}$ & 3.658815 & -2.434037 & -0.940370 \\
$\mathrm{C}$ & 5.783693 & 0.125508 & -0.208844 \\
$\mathrm{H}$ & 5.215788 & 2.053509 & 0.518469 \\
$\mathrm{H}$ & 6.063512 & -1.893394 & -0.946906 \\
$\mathrm{H}$ & 6.844786 & 0.353153 & -0.215410 \\
\hline
\end{tabular}

Table S14. Cartesian coordinates of the optimized geometry of BNp cation computed at CAM$\mathrm{B} 3 \mathrm{LYP} / 6-31+\mathrm{G}(\mathrm{d}, \mathrm{p})$ level of theory (charge $=+1$; spin multiplicity $=2, \mathrm{E}=-769.912642972$, number of imaginary frequencies $=0$ ).

\begin{tabular}{llll}
\hline Atom & $\mathrm{X}$ & $\mathrm{Y}$ & $\mathrm{Z}$ \\
\hline $\mathrm{C}$ & -5.355205 & 1.189105 & -0.403075 \\
$\mathrm{C}$ & -4.020638 & 1.511684 & -0.375260 \\
$\mathrm{C}$ & -3.044519 & 0.509855 & -0.109322 \\
$\mathrm{C}$ & -3.465802 & -0.834802 & 0.121209 \\
$\mathrm{C}$ & -4.832977 & -1.134952 & 0.087302 \\
$\mathrm{C}$ & -5.761865 & -0.138976 & -0.170387 \\
$\mathrm{H}$ & -1.377092 & 1.832807 & -0.272464 \\
$\mathrm{H}$ & -6.098541 & 1.952116 & -0.604409 \\
$\mathrm{H}$ & -3.694913 & 2.531706 & -0.553627 \\
$\mathrm{C}$ & -1.679826 & 0.812127 & -0.068977 \\
$\mathrm{C}$ & -2.468164 & -1.827685 & 0.378517 \\
$\mathrm{H}$ & -5.161643 & -2.154064 & 0.263653 \\
$\mathrm{H}$ & -6.818083 & -0.385614 & -0.194221 \\
$\mathrm{C}$ & -1.143835 & -1.518050 & 0.394410
\end{tabular}




\begin{tabular}{llll}
$\mathrm{C}$ & -0.699861 & -0.175569 & 0.172581 \\
$\mathrm{H}$ & -2.789295 & -2.846055 & 0.573343 \\
$\mathrm{H}$ & -0.424721 & -2.293332 & 0.625652 \\
$\mathrm{C}$ & 0.699862 & 0.175562 & 0.172587 \\
$\mathrm{C}$ & 1.679831 & -0.812134 & -0.068962 \\
$\mathrm{C}$ & 1.143831 & 1.518044 & 0.394415 \\
$\mathrm{C}$ & 3.044522 & -0.509856 & -0.109315 \\
$\mathrm{H}$ & 1.377099 & -1.832814 & -0.272449 \\
$\mathrm{C}$ & 2.468160 & 1.827681 & 0.378523 \\
$\mathrm{H}$ & 0.424715 & 2.293328 & 0.625642 \\
$\mathrm{C}$ & 3.465799 & 0.834803 & 0.121207 \\
$\mathrm{C}$ & 4.020643 & -1.511680 & -0.375261 \\
$\mathrm{H}$ & 2.789288 & 2.846052 & 0.573350 \\
$\mathrm{C}$ & 4.832973 & 1.134956 & 0.087302 \\
$\mathrm{C}$ & 5.355208 & -1.189097 & -0.403081 \\
$\mathrm{H}$ & 3.694922 & -2.531703 & -0.553632 \\
$\mathrm{C}$ & 5.761864 & 0.138982 & -0.170387 \\
$\mathrm{H}$ & 5.161634 & 2.154069 & 0.263652 \\
$\mathrm{H}$ & 6.098544 & -1.952106 & -0.604423 \\
$\mathrm{H}$ & 6.818081 & 0.385624 & -0.194225 \\
\hline
\end{tabular}

4. X-ray crystallographic data and structure refinement for all the target compounds

Table S15. Crystal data and structure refinement for BAz1 and BAz2.

\begin{tabular}{ccc}
\hline Compound & $\mathrm{BAz} 1$ & $\mathrm{BAz2}$ \\
\hline Chemical formula & $\mathrm{C}_{20} \mathrm{H}_{14}$ & $\mathrm{C}_{20} \mathrm{H}_{14}$ \\
Formula weight & 254.31 & 254.31 \\
Temperature (K) & $296 \mathrm{~K}$ & $296 \mathrm{~K}$ \\
Radiation type & $\mathrm{Cu} \mathrm{K \alpha}$ & Mo $K \alpha$ \\
Crystal system & Monoclinic & Triclinic \\
Space group & $P 2_{1} / \mathrm{c}$ & $P-1$ \\
$a(\AA)$ & $14.0565(7)$ & $5.9457(3)$ \\
$b(\AA)$ & $5.9201(3)$ & $7.8815(5)$ \\
$c(\AA)$ & $7.8474(4)$ & $14.0822(8)$ \\
$\alpha\left({ }^{\circ}\right)$ & 90 & $86.684(5)$ \\
$\beta\left({ }^{\circ}\right)$ & $92.837(3)$ & $87.090(5)$
\end{tabular}


$\gamma\left({ }^{\circ}\right)$

Volume $\left(\AA^{3}\right)$

Z

Density (calculated) $\left(\mathrm{g} / \mathrm{cm}^{3}\right)$

Absorption coefficient $\left(\mathrm{mm}^{-1}\right)$

$F(000)$

Crystal size (mm)

Theta range for data collection $\left({ }^{\circ}\right)$

Index ranges

Reflections collected

Independent reflections

Completeness (\%)

Absorption correction

Maximum and minimum transmissions

Refinement method

Goodness-of-fit on $F^{2}$

Final $R$ indices $[I>2 \sigma(I)]$

$R$ indices (all data)

Largest difference peak and hole $\left(\mathrm{e} \cdot \AA^{-3}\right)$
$88.848(5)$

652.23

2

$657.85(7)$

2

1.295

1.284

0.554

0.073

268

268

$0.13 \times 0.09 \times 0.03$

$0.16 \times 0.08 \times 0.03$

3.148 to 68.222

$-16 \leq h \leq 16$

2.895 to 27.496

$-7 \leq k \leq 7$

$-7 \leq h \leq 7$

$-10 \leq k \leq 10$

$-9 \leq l \leq 9$

11264

$1191\left[R_{\text {int }}=0.0983\right]$

$-18 \leq l \leq 18$

8859

$100.0\left(\theta=25.00^{\circ}\right)$

Multi scan

1.000 and 0.733

$\left.R_{\text {int }}=0.0551\right]$

$99.3\left(\theta=25.242^{\circ}\right)$

Multi scan

1.000 and 0.797

Full-matrix least-squares on Full-matrix least-squares on

$F^{2}$

$F^{2}$

$1191 / 138 / 181$

$2996 / 0 / 181$

0.905

1.054

$R_{1}=0.0958$

$R_{1}=0.0583$

$w R_{2}=0.2527$

$w R_{2}=0.1522$

$R_{1}=0.1901$

$R_{1}=0.1123$

$w R_{2}=0.3442$

$w R_{2}=0.1522$

0.195 and -0.210

0.161 and -0.152

CCDC number

1951595

1951596

Table S16. Crystal data and structure refinement for BAz3 and BNp.

\begin{tabular}{ccc}
\hline Compound & $\mathrm{BAz3}$ & $\mathrm{BNp}$ \\
\hline Chemical formula & $\mathrm{C}_{20} \mathrm{H}_{14}$ & $\mathrm{C}_{20} \mathrm{H}_{14}$ \\
Formula weight & 254.31 & 254.31 \\
Temperature (K) & $296 \mathrm{~K}$ & $293 \mathrm{~K}$ \\
Radiation type & $\mathrm{Cu} K \alpha$ & Mo $K \alpha$ \\
Crystal system & Triclinic & Monoclinic \\
Space group & $P-1$ & $P 2_{1} / \mathrm{c}$ \\
$a(\AA)$ & $6.0870(2)$ & $13.5349(7)$
\end{tabular}


$b(\AA)$

$c(\AA)$

$\alpha\left(^{\circ}\right)$

$\beta\left(^{\circ}\right)$

$\gamma\left({ }^{\circ}\right)$

Volume $\left(\AA^{3}\right)$

Z

Density (calculated) $\left(\mathrm{g} / \mathrm{cm}^{3}\right)$

Absorption coefficient $\left(\mathrm{mm}^{-1}\right)$

$F(000)$

Crystal size (mm)

Theta range for data collection $\left(^{\circ}\right)$

Index ranges

Reflections collected

Independent reflections

Completeness (\%)

Absorption correction

Maximum and minimum transmissions

Refinement method

Goodness-of-fit on $F^{2}$

Final $R$ indices $[I>2 \sigma(I)]$

$R$ indices (all data)

Largest difference peak and hole $\left(\mathrm{e} \cdot \AA^{-3}\right)$
$7.5639(3)$

14.4293(5)

97.942(2)

95.597(2)

90.921(3)

654.54(4)

2

1.290

0.552

268

$0.15 \times 0.09 \times 0.03$

6.282 to 67.497

$-7 \leq h \leq 7$

$-9 \leq k \leq 9$

$-17 \leq l \leq 16$

11019

$2321\left[R_{\text {int }}=0.0362\right]$

$98.3\left(\theta=67.497^{\circ}\right)$

Multi scan

1.000 and 0.887
6.0042(4)

8.1543(4)

90

94.232(4)

90

660.86(6)

2

1.278

0.072

268

$0.15 \times 0.10 \times 0.05$

3.714 to 27.495

$-17 \leq h \leq 17$

$-7 \leq k \leq 7$

$-10 \leq l \leq 10$

8657

$1514\left[R_{\text {int }}=0.0400\right]$

$99.1\left(\theta=25.242^{\circ}\right)$

Multi scan

1.000 and 0.687

Full-matrix least-squares on Full-matrix least-squares on

$F^{2}$

$2321 / 0 / 181$

1.157

$R_{1}=0.0492$

$w R_{2}=0.1129$

$R_{1}=0.0602$

$w R_{2}=0.1286$

0.220 and -0.344
$F^{2}$

$1514 / 0 / 91$

1.080

$R_{1}=0.0527$

$w R_{2}=0.1404$

$R_{1}=0.0712$

$w R_{2}=0.1550$

0.241 and -0.173 


\section{FET characteristics of biazulene isomers}
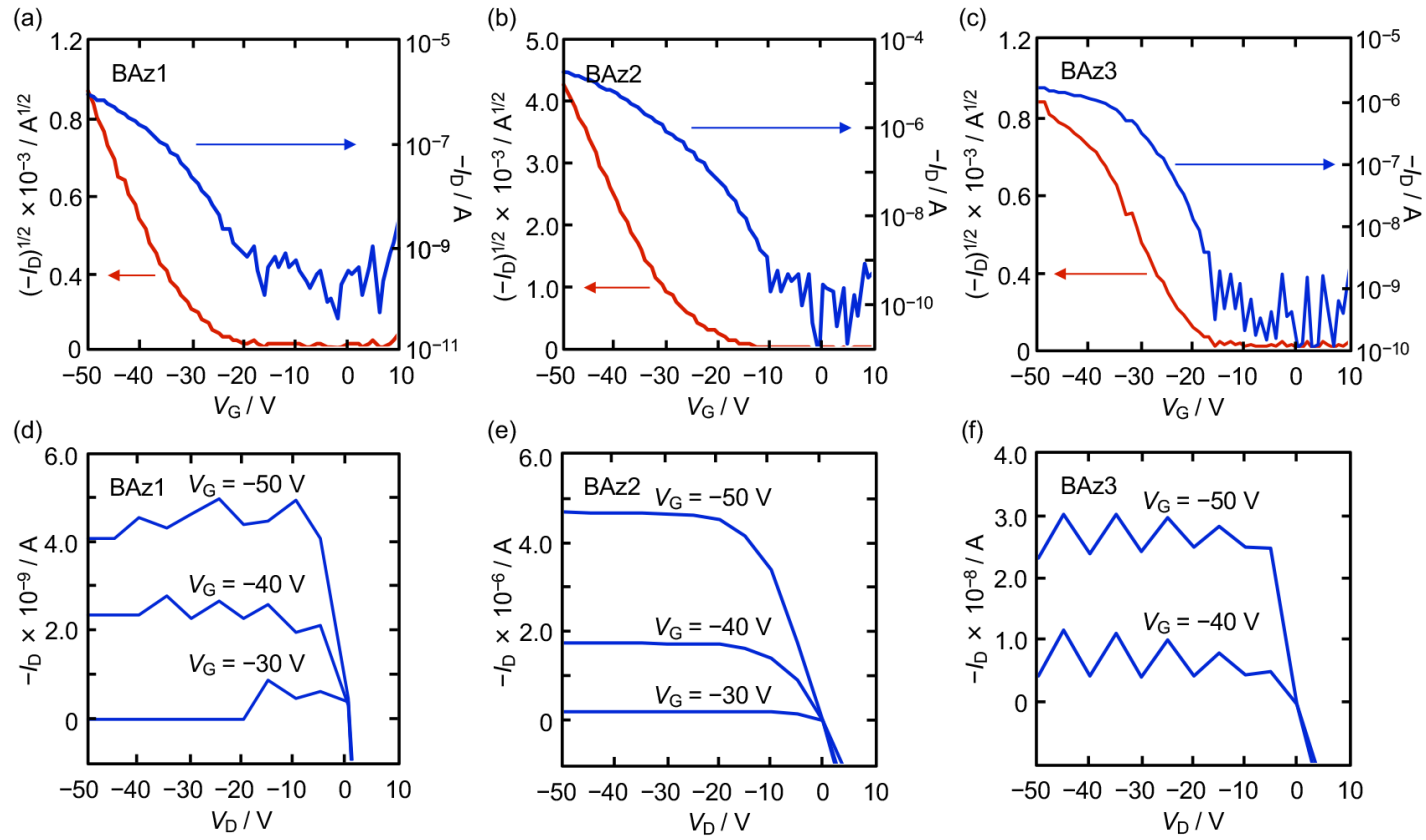

Figure S5. Typical OFET characteristics of top-contact bottom-gate devices of BAz1, BAz2 and BAz3 thin film fabricated by vacuum deposition. Transfer curves in the saturated region (a-c) and output curves at different gate voltages (d-f).
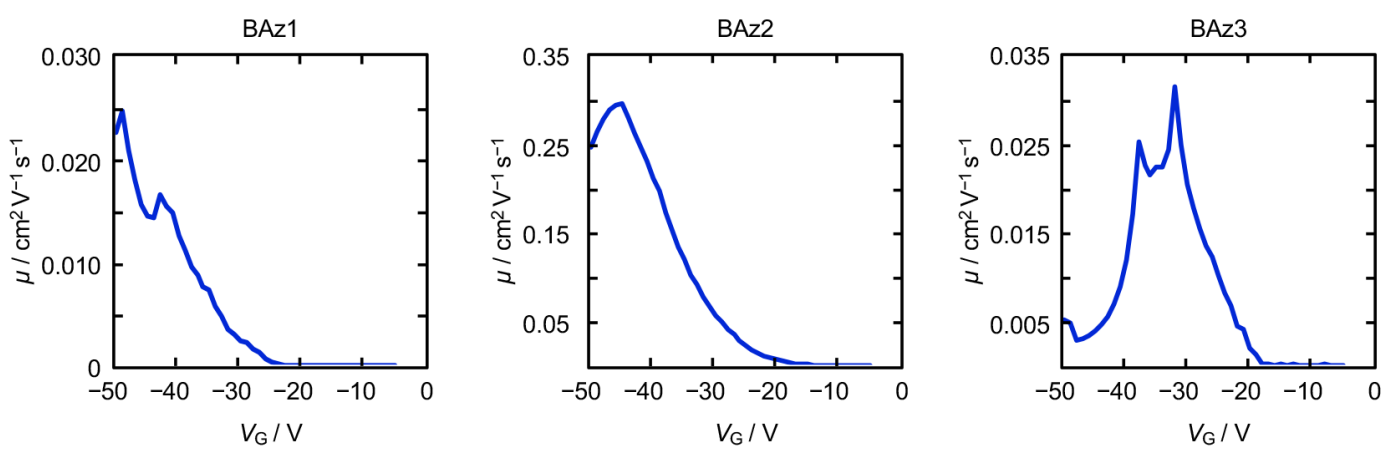

Figure S6. Mobility as a function of $V_{\mathrm{G}}$ in OFET devices of BAz1, BAz2 and BAz3 thin film. 

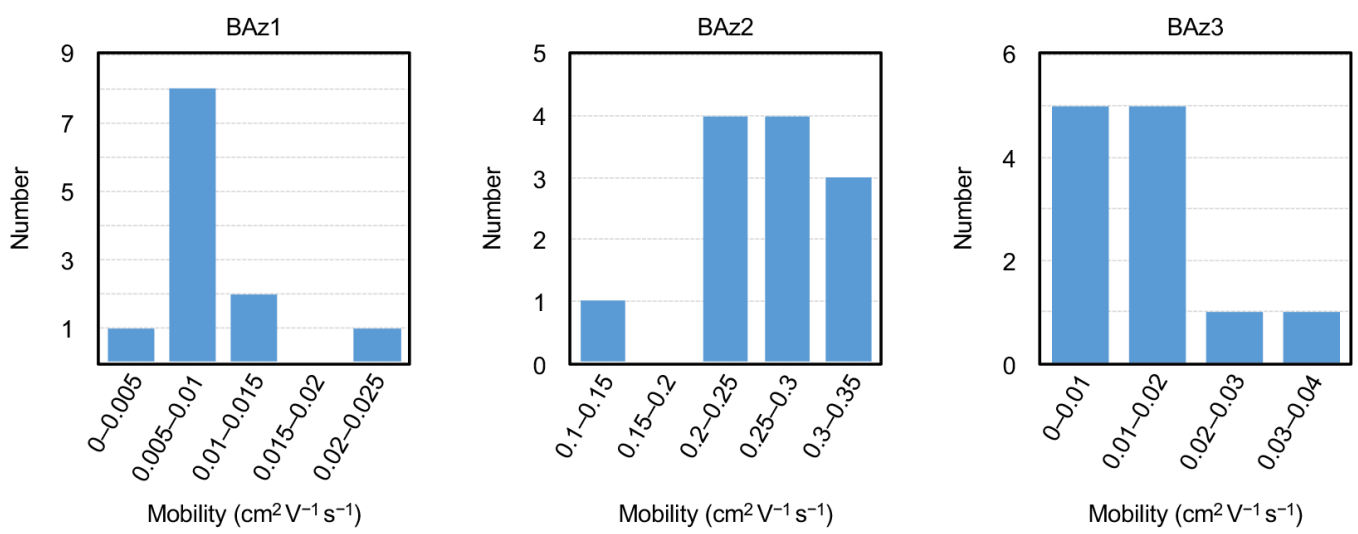

Figure S7. Histogram of the mobility distributions obtained from 12 OFET devices of BAz1, BAz2 and BAz3 thin film. 